\title{
Distributed sensitivity for movement amplitude in directionally tuned neuronal populations
}

\section{Citation}

Fabbri, S., A. Caramazza, and A. Lingnau. 2011. “Distributed Sensitivity for Movement Amplitude in Directionally Tuned Neuronal Populations." Journal of Neurophysiology 107 (7) (December 28): 1845-1856. doi:10.1152/jn.00435.2011.

\section{Published Version}

doi:10.1152/jn.00435.2011

\section{Permanent link}

http://nrs.harvard.edu/urn-3:HUL.InstRepos:33719901

\section{Terms of Use}

This article was downloaded from Harvard University's DASH repository, and is made available under the terms and conditions applicable to Open Access Policy Articles, as set forth at http:// nrs.harvard.edu/urn-3:HUL.InstRepos:dash.current.terms-of-use\#OAP

\section{Share Your Story}

The Harvard community has made this article openly available.

Please share how this access benefits you. Submit a story.

Accessibility 


\section{Distributed sensitivity for movement amplitude in directionally-tuned neuronal populations}

$9 \quad{ }^{1}$ Center for Mind/ Brain Sciences (CIMeC), University of Trento, 38100 Mattarello, Italy

$10{ }^{2}$ Department of Psychology, Harvard University, Cambridge, MA 02138, USA

13 Running head: Sensitivity for movement amplitude

$16{ }^{*}$ Corresponding author

17 Angelika Lingnau, angelika.lingnau@unitn.it

18 Center for Mind/Brain Science - CIMeC

19 University of Trento,

20 Via delle Regole 101, 38060 Mattarello (TN)

21 Italy

22 Tel. +390464808664

$23 \quad$ Tel. +390461883067 


\section{Abstract}

26 Neurons in macaque primary motor cortex (M1) and dorsal premotor cortex (PMd) are

27 tuned to movement direction. In humans, neuronal populations tuned to movement

28 direction have recently been described using multi-voxel pattern analysis and functional

29 magnetic resonance imaging (fMRI) adaptation. It is unclear, however, to what extent

30 directionally-tuned neuronal populations are sensitive to movement amplitude. Here we

31 used fMRI adaptation to determine whether directionally-tuned neuronal populations are

32 modulated by movement amplitude. In different blocks, participants were adapted to

33 small or large amplitude hand reaching movements. On occasional test trials, we

34 parametrically varied the angular difference between adaptation and test direction and the

35 congruency between adapted and tested amplitude (same or different). We predicted that

36 the blood oxygen level dependent (BOLD) signal in directionally-tuned regions should be

37 adapted in proportion to the angular difference between adaptation and test direction.

38 Directionally-tuned regions insensitive to movement amplitude should show a transfer of

39 adaptation from the adapted to the non-adapted amplitude. In contrast, regions sensitive

40 to the specific combination of movement direction and amplitude should show directional

41 tuning only for the adapted amplitude. We identified a network of parietal and frontal

42 regions tuned to movement direction. We found that parietal areas contain neuronal

43 populations sensitive to specific combinations of movement direction and amplitude,

44 while frontal areas show transfer from the adapted to the non-adapted amplitude during

45 small amplitude movements after adaptation to large amplitude, but not vice versa. Our 
46 results thus imply different processing of movement amplitude in directionally tuned

47 frontal and parietal areas.

48 Keywords: directional tuning, fMRI adaptation, movement direction, movement extent 


\section{Introduction}

50 To be able to reach objects, both movement direction and amplitude need to be specified.

51 An extensive literature from monkey physiology reported directional tuning in many

52 regions of the monkey brain (Caminiti et al. 1991; Fortier et al. 1989; Georgopoulos et al.

53 1982; Kakei et al. 2001; Kalaska et al. 1983).

54 Less is known about the representation of movement amplitude. Neurophysiological

55 studies suggest that many neurons in M1 and PMd are sensitive to both movement

56 direction and amplitude, but only small proportions of cells seem to be sensitive to

57 movement amplitude alone (Fu et al. 1995; Fu et al. 1993; Kurata 1993; Messier and

58 Kalaska 2000; Riehle and Requin 1989). Moreover, it has been suggested that the

59 importance of direction and amplitude varies with time, with sensitivity to movement

60 amplitude increasing the closer it gets to the execution of the movement (Messier and

61 Kalaska 2000). It is unknown, however, whether directionally-tuned areas beyond M1

62 and PMd are sensitive to movement amplitude, and if so, whether there are qualitative

63 differences between these regions.

64 In humans, many neuroimaging studies investigated the neuronal basis of reaching 65 movements (Cavina-Pratesi et al. 2010; Connolly et al. 2007; Culham et al. 2003;

66 Desmurget et al. 2001; Filimon et al. 2007; Frey et al. 2005). Both parietal (Fernandez-

67 Ruiz et al. 2007; Van Der Werf et al. 2010) and frontal areas (Beurze et al. 2009) have 
68 been shown to be sensitive to the difference between movements directed in ipsi- versus

69 contralateral space. It is unclear, however, if these results reflect a more general

70 preference for movements directed towards the space contralateral to the hand, or if these

71 areas contain neuronal populations that are tuned to movement direction

72 In humans, the representation of movement direction and amplitude are difficult to

73 examine due to the limited spatial resolution of non-invasive techniques such as

74 functional magnetic resonance imaging (fMRI). With the emergence of more

75 sophisticated methods such as fMRI-Adaptation (Grill-Spector and Malach 2001) and

76 multi-voxel pattern analysis (Haxby et al. 2001), sensitivity to movement direction in the

77 human motor system has recently been demonstrated in M1 (Eisenberg et al. 2010) as

78 well as in several additional premotor and parietal areas (Fabbri et al. 2010). It is

79 unknown, however, whether neuronal populations in these areas are sensitive to

80 movement direction only, irrespective of movement amplitude, or whether they are tuned

81 to specific combinations of movement direction and amplitude.

82 Here we address this question using an fMRI adaptation paradigm similar to our previous

83 study (Fabbri et al. 2010). We instructed participants to execute reaching movements

84 with a constant direction $\left(90^{\circ}\right)$ and amplitude (6 or $12 \mathrm{~cm}$; see Figure 1a, b). After an

85 adaptation sequence of 3 to 8 trials, participants were instructed to execute a test reaching

86 movement that varied with respect to the adapted direction by $0^{\circ},+/-45^{\circ}$ or $+/-90^{\circ}$ 
87 (Figure 1b, c). In half of these test trials, movement amplitude was kept constant (adapt 88 small, test small/ adapt large, test large), while in the other half of the test trials

89 movement amplitude varied (adapt small, test large/ adapt large, test small). We predicted

90 that the BOLD signal within voxels containing directionally-tuned neurons would adapt

91 maximally during movements with $0^{\circ}$ angular difference between adaptation and test

92 direction and show a recovery from adaptation in relation to the angular difference

93 between adaptation and test direction, similar to our previous findings (Fabbri et al.

94 2010). Furthermore we reasoned that neuronal populations that represent movement

95 direction irrespective of movement amplitude should adapt to movement direction both

96 for adapted and non-adapted amplitudes (see Figure 1d). By contrast, within

97 directionally-tuned neuronal populations that are sensitive both to movement direction

98 and amplitude, we should find no transfer of adaptation to movement direction from the

99 adapted to the non-adapted amplitude (see Figure 1e).

101 We identified several areas within the human visuomotor system that showed directional

102 tuning, similar to our previous results. Parietal areas showed no transfer from the adapted

103 to the non-adapted amplitude, suggesting that these neuronal populations are tuned to

104 specific combinations of movement direction and amplitude. Frontal areas, by contrast, 105 showed a partial transfer from the adapted to the non-adapted amplitude when 
106 participants were adapted to the large amplitude and tested with the small amplitude, but

107 not vice versa. We discuss several alternative neural mechanisms that might underlie this

108 asymmetry.

109 


\section{Materials and Methods}

\section{Participants}

112 Fourteen volunteers (6 males) took part in the Experiment (mean age 27; range, 23-35

113 years). All participants were right handed. Vision was normal or corrected-to-normal

114 using MR-compatible glasses. All participants were naïve to the purpose of the study;

115 they were neurologically intact and gave written informed consent for their participation.

116 The experimental procedures were approved by the Ethical Committee for Research

117 Involving Human Subjects at the University of Trento.

\section{Procedure and Visual Stimulation}

119 During each trial, participants were presented with an arrow at the center of the screen for

1202 seconds (s), followed by an inter-trial-interval (ITI) of $1 \mathrm{~s}$ (see Figure 1c). Participants

121 had to execute a center-out reaching task on a device attached to their chest, using their

122 right hand (see Figure 1a). The device consisted of 11 half-spheres of polystyrene $(3 \mathrm{~cm}$

123 diameter) glued on a black plastic surface $(15 \times 27,5 \mathrm{~cm})$. The half-spheres were arranged

124 on two concentric semicircles (6 and $12 \mathrm{~cm}$ radius) of 5 half-spheres each and 1 at the

125 common center.

126 At the beginning of each trial, participants positioned their index finger on the central

127 half-sphere (see Figure 1a). As soon as the arrow appeared on the screen, participants

128 started the reaching movement on the device in the direction indicated by the orientation

129 of the arrow. The color of the arrow specified the amplitude of the movement: a red

130 arrow instructed participants to reach towards a target on the near semicircle (small 8 
131 amplitude); a blue arrow instructed participants to reach towards targets on the far 132 semicircle (large amplitude).

133 Since we observed no qualitative differences between two different adaptation directions

134 in our previous study (Fabbri et al. 2010), we used one adaptation direction, central ahead

135 (see Figure 1b), in the current experiment. In half of the scanning runs, participants were

136 adapted to small amplitude reaching movements; in the other half, they were adapted to

137 large amplitude reaching movements. The sequence of scanning runs was alternated

138 between small and large adaptation amplitudes within participants (e.g. ABAB), and the

139 order was counterbalanced between participants (e.g. participant 1, ABAB vs. participant

140 2, BABA).

141 During the adaptation sequence, the same direction was repeated from 3 to 8 trials in 142 order to reduce predictability of test trial occurrence. After each sequence of adaptation 143 trials, a test trial was presented (Figure 1c). During test trials, we parametrically varied 144 the angular difference between adaptation and test directions $\left(0^{\circ},+/-45^{\circ},+/-90^{\circ}\right)$ as well 145 as movement amplitude (small, large) (see Figure 1b).

146 To ensure that any signs of directional sensitivity are due to adaptation to movement 147 direction and not due to the repetition of low-level perceptual features of the arrows, we 148 varied the visual appearance of the arrow that indicated the movement direction and 149 movement amplitude on each trial (see Fabbri et al. 2010 for a similar approach). Arrow 150 width and length was varied randomly from $0.51^{\circ}$ to $1.51^{\circ}$ in steps of $0.25^{\circ}$. The $\mathrm{x}$ - and $151 \mathrm{y}$ - center coordinates of the arrow were jittered in a range of $+/-0.07^{\circ}$ in steps of $0.035^{\circ}$. 9 
152 Stimuli were back-projected onto a screen by a liquid-crystal projector at a frame rate of

$15360 \mathrm{~Hz}$ and a screen resolution of $1,280 \times 1,024$ pixels (mean luminance: $109 \mathrm{~cd} / \mathrm{m}^{2}$ ).

154 Participants viewed the stimuli binocularly through a mirror above the head coil. The

155 screen was visible as a rectangular aperture of $17.5 \times 14.3$ degrees.

156 Visual stimulation was controlled by ASF (Schwarzbach 2011) based on the MATLAB

157 Psychtoolbox-3 for Windows (Brainard 1997; Pelli 1997).

\section{Instructions and Training}

159 Before entering the scanner, participants learned to execute the reaching movements

160 corresponding to the visual instructions, and they familiarized themselves with the

161 location of the half spheres on the device such that they were able to perform accurate

162 reaching movements in the absence of visual feedback. The experimenter asked

163 participants to execute every reaching movement within a constant time window of $2 \mathrm{~s}$

164 corresponding to the presentation time of the arrow, rather than trying to move as fast as

165 possible and thus risking head movements. Participants were asked to move their hand

166 back to the center position before the arrow disappeared, and to start each trial from the

167 center position.

168 fMRI Adaptation Design

169 The entire experiment consisted of 12 event-related fMRI adaptation runs. Each run

170 consisted of 78 trials (66 adaptation trials plus 12 test trials) and lasted 4.9 minutes. 
171 In each run, test trials with angular difference $+/-45^{\circ}$ and $+/-90^{\circ}$ were repeated once for

172 each test amplitude (small, large). Since we initially intended to collapse across test

173 directions to the left (-) and right $(+)$ of the adaptation direction in the analysis, we had

174 two repetitions for each absolute angular difference: $45^{\circ}$ and $90^{\circ}$ for both test amplitudes.

175 In order to have the same number of repetitions for each test direction, test trials with $0^{\circ}$

176 angular difference were repeated twice for each test amplitude. Thus, there were 6 test

177 trials for each test amplitude, for a total of 12 test trials per run.

178 There were 3 to 8 adaptation trials between two successive test trials, resulting in 6

179 different adaptation intervals, amounting to $33(3+4+5+6+7+8)$ adaptation trials. Each

180 adaptation interval was repeated twice resulting in 12 adaptation intervals and 66

181 adaptation trials per run. The number of adaptation trials between two successive test

182 trials was randomly assigned to each condition.

183 To minimize muscle fatigue related to the task, breaks of $20 \mathrm{~s}$ were inserted after half a

184 run (i.e., after $2.45 \mathrm{~min}$ ). Trials in both the first and the second half of each run consisted

185 of 6 test trials, each following one of the randomly distributed 6 adaptation intervals

186 giving a total of 39 trials (33 adaptation trials +6 test trials) per half run. In one half of

187 the run, each test direction was repeated once, with the exception of $0^{\circ}$ angular

188 difference, which was repeated twice, for a total of 6 test trials. Each test direction was

189 paired with one test amplitude in one half of the run and with the other test amplitude in

190 the other half of the run. Since both test amplitudes appeared in each half run, half of the

191 test directions were paired with one test amplitude and the other directions were paired 11 
192 with the other test amplitude. The assignment was reversed in the other half run. The only

193 exception was $0^{\circ}$ angular difference, which was paired with both test amplitudes in each

194 half run.

\section{Data Acquisition}

196 We acquired fMRI data using a 4T Bruker MedSpec Biospin MR scanner and an 8-

197 channel birdcage head coil. Functional images were acquired with a T2*-weighted

198 gradient-recalled echo-planar imaging (EPI) sequence. Before each functional scan, we

199 performed an additional scan to measure the point-spread function (PSF) of the acquired

200 sequence, which serves for correction of the distortion expected with high-field imaging

201 (Zaitsev et al. 2004). We used 34 slices, acquired in ascending interleaved order, slightly

202 tilted to run parallel to the calcarine sulcus (TR (time to repeat): $2000 \mathrm{~ms}$; voxel

203 resolution: $3 \times 3 \times 3 \mathrm{~mm}$; TE (echo time): $33 \mathrm{~ms}$; flip angle (FA): $73^{\circ}$; field of view (FOV):

204192 x $192 \mathrm{~mm}$; gap size: $0.45 \mathrm{~mm}$ ). Each participant completed 12 scans of 147 volumes

205 each.

206 To be able to co-register the low-resolution functional images to a high-resolution

207 anatomical scan, we acquired a T1 weighted anatomical scan (MP-RAGE; voxel

208 resolution: 1x 1 x 1 mm; FOV: 256 × 224 mm; GRAPPA acquisition with an acceleration

209 factor of 2; TR: $2700 \mathrm{~ms}$, inversion time (TI), $1020 \mathrm{~ms}$; FA: $7^{\circ}$ ).

\section{Data Analysis}

211 Data analysis was performed using BrainVoyager QX 2.1 (Brain Innovation) and custom

212 software written in MATLAB (Mathworks). Data recorded from one participant were 12 
213 excluded from the analysis because of several abrupt head movements, as was evident

214 from the first derivative of the 3D motion correction parameters.

215 Preprocessing, segmentation, and flattening. To correct for distortions in geometry

216 and intensity in the EPI images, we applied distortion correction on the basis of the PSF

217 data acquired before each EPI scan (Zeng and Constable 2002). Before further analysis,

218 we removed the first 4 volumes to avoid T1-saturation. Next, we performed 3D motion

219 correction with trilinear interpolation using the first volume as reference followed by

220 slice timing correction with ascending interleaved order. Functional data were temporally

221 high-pass filtered using a cut-off frequency of 3 cycles per run as well as linear trend

222 removal. The time course of each voxel was normalized to reflect percent signal change

223 using the following formula:

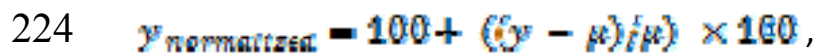

226 where $\gamma$ equals the observed time course of a single voxel, and $\mu$ equals the mean of the

227 observed time course of that voxel.

228 We applied spatial smoothing with a Gaussian kernel of $8 \mathrm{~mm}$ full width at half

229 maximum. Next, we aligned the first volume of each run to the high resolution anatomy.

230 Both functional and anatomical data were transformed into Talairach space using trilinear

231 interpolation. 
232 Definition of Regions of Interest (ROIs). To identify our regions of interest, we ran a

233 massive univariate random effects (RFX) general linear model (GLM) analysis. In brief,

234 for each voxel the GLM analysis of fMRI data is carried out to fit a model for that 235 voxel's predicted time course. The predicted time course is based on the timing of the 236 experimental conditions and the expected shape of the hemodynamic response function.

237 The basic formula for this regression analysis is given by

$238 y=\beta X+a$,

239 where $\gamma$ is the observed time course of a single voxel, $\mathrm{x}$ is the design matrix with the

240 different predictors, and $\beta$ is a vector of the to be estimated weights for each of the

241 predictors, and $\boldsymbol{\varepsilon}$ is the residual noise in the data. The GLM finds those weights that

242 minimize the residual error.

243 To model our experimental conditions, we included the factors adaptation amplitude

244 (small, large), angular difference between adaptation and test direction $\left(0^{\circ},+/-45^{\circ},+/-\right.$

$24590^{\circ}$ ), and test amplitude (small, large). Altogether, this resulted in the following 22

246 predictors:

247 adapt small;

248 adapt large; 
249 test small preceded by adapt small, angular difference $-90^{\circ} /-45^{\circ} / 0^{\circ} /+45^{\circ} /+90^{\circ}$;

250 test large preceded by adapt small, angular difference $-90^{\circ} /-45^{\circ} / 0^{\circ} /+45^{\circ} /+90^{\circ}$;

251 test small preceded by adapt large, angular difference $-90^{\circ} /-45^{\circ} / 0^{\circ} /+45^{\circ} /+90^{\circ}$; and

252 test large preceded by adapt large, angular difference $-90^{\circ} /-45^{\circ} / 0 \%+45^{\circ} /+90^{\circ}$.

253 In addition to the predictors for our experimental conditions, we included the first and

254 second derivatives of each predictor time course in order to be able to model shift and

255 dispersion of the hemodynamic impulse response function, respectively. Moreover, six

256 additional parameters resulting from 3D motion correction $(\mathrm{x}, \mathrm{y}, \mathrm{z}$ rotation and

257 translation) were included in the model. The first and second derivatives and the 3D

258 motion correction parameters were included as predictors of no interest.

259 Each predictor time course was convolved with a dual-gamma hemodynamic impulse

260 response function (Friston et al. 1998), and the resulting reference time courses were used

261 to fit the signal time course of each voxel. For each predictor and each voxel, this

262 analysis resulted in one GLM parameter estimate (beta weight) for the amplitude of the

263 BOLD signal. Since the time course of each voxel was normalized to reflect percent

264 signal change, the beta estimates provide an estimate of percent signal change. We

265 submitted the individually estimated beta weights to a second level analysis with

266 participants as a random factor, which yields a random-effects (RFX) analysis.

267 In order to identify areas sensitive to a change in movement direction or test amplitude,

268 and to avoid selection of ROIs biased in favor of our hypothesis on movement selectivity 15 
269 (Kriegeskorte et al. 2009), we functionally selected ROIs by computing the RFX GLM

270 contrast test trials "adapted direction, adapted amplitude" versus all remaining test trials.

271 Statistical maps were corrected for multiple comparisons using a False Discovery Rate

272 (FDR) $<.005$ with a cluster threshold of 4 voxels.

273 In order to compare results between different regions, we selected spherical ROIs with a

274 diameter of $5 \mathrm{~mm}$ (515 voxels) around the center of gravity of each region using the plug-

275 in "Talairach coordinates to VOI" available from BrainInnovation, The Netherlands. The

276 resulting activations were plotted on the anatomy of the MNI Template Brain "Colin 27"

277 (Van Essen 2002).

278 Statistical analysis. To quantify the effect of the angular difference between adaptation

279 and test directions as well as the effect of test amplitude, we extracted non-standardized

280 beta estimates of the amplitude of the BOLD response resulting from the RFX GLM

281 analysis described above in each ROI for each of the 5 angular differences between

282 adaptation and test direction $\left(-90^{\circ},-45^{\circ}, 0^{\circ},+45^{\circ},+90^{\circ}\right)$, separately for the two

283 adaptation and test amplitudes. Next, we submitted these beta estimates to a 2

284 (Adaptation Amplitude) x 2 (Test Amplitude) x 5 (Angular Difference Between

285 Adaptation and Test Direction) repeated-measures ANOVA, separately for each ROI. To

286 examine directional tuning separately for the two adaptation amplitudes, we conducted

287 two additional ANOVAs with factors Test Amplitude and Angular Difference, separately

288 for small and large adaptation amplitudes. Finally, to quantify directional tuning, we 
289 carried out complex contrasts for the factor Angular Difference [ [ $\left.\begin{array}{lllll}2 & 1 & -6 & 1 & 2\end{array}\right]$, separately

290 for each combination of adaptation and test amplitude in each ROI.

\section{Kinematics}

293 To measure spatial and temporal accuracy of the reaching movements, we carried out an

294 additional control experiment with 5 participants outside of the scanner using the same 295 setup and design as during the fMRI session.

296 Participants were seated $70 \mathrm{~cm}$ in from of a screen, with the device placed $30 \mathrm{~cm}$ in front 297 of them on a table. They were instructed to position their head in a chin rest. A black 298 plastic surface positioned below the participants' chins prevented vision of the hand and 299 the device.

300 To examine reaching kinematics, we attached a reflective marker to the tip of the right 301 index finger of the participants and recorded the position of the marker with a movement 302 analysis system (Qualisys) at $250 \mathrm{~Hz}$. The onset of each trial was indicated by a voltage 303 change sent from the stimulation PC via the parallel port to an analog board that was

304 connected to the movement analysis system. In a separate block, we attached markers to 305 the position of each of the 10 target positions and the central start position. These points 306 served as reference for data analysis. After one run of training during which the 307 participants became familiar with the device and the task, we collected data from 12 runs, 308 using the same stimuli and number of repetitions as in the fMRI experiment. 
309 To quantify temporal accuracy, we computed the time between the onset of the trial and

310 the end of the movement from the target back to the central target position. The latter

311 time point was determined by first computing the time of the peak of the movement

312 velocity towards the target as well as the peak from the target back towards the central

313 target position and then identifying the time point after the second peak at which

314 movement velocity dropped below $10 \mathrm{~cm} / \mathrm{s}$.

315 To quantify spatial accuracy, we first computed the peak of the movement towards the

316 target as well as the peak from the target back towards the central target position. We

317 then determined the time of the trough between the two peaks and computed the standard

318 deviation of the horizontal and vertical distance between the index finger and the center

319 coordinate of the target location at the time of the trough (see Brouwer et al. 2000, for a

320 similar approach).

321 To test whether movement velocity co-varied with movement amplitude in our

322 experiment, we computed the peak velocity for the movement from the central start

323 position towards the target. 


\section{Results}

\section{Areas involved during hand reaching movements}

327 Our first aim was to identify regions of interest (ROIs) that were sensitive to a change in

328 movement direction or amplitude, as revealed by the contrast between test trials that

329 differed from adaptation trials and test trials that were identical to adaptation trials. Areas

330 that were revealed by this contrast are shown in Figure $\mathbf{2}$ and consisted of bilateral

331 insula, ventral premotor cortex (PMv), supplementary motor area (SMA), dorsal

332 premotor cortex (PMd), left M1, bilateral inferior parietal lobule (IPL), anterior and

333 posterior intraparietal sulcus (aIPS and pIPS, respectively), right anterior precuneus

$334(\mathrm{aPCu})$ and bilateral superior parieto-occipital cortex (SPOC). An overview of the

335 Talairach coordinates of these areas can be found in Table 1.

$336<<$ Add Figure 2 here $>>$

$337<<$ Add Table 1 here $>>$

\section{Sensitivity for movement direction and amplitude}

339 In our previous study, we identified directionally-tuned neuronal populations in several

340 regions of the human visuomotor system: the BOLD signal adapted maximally for

341 movements in the same direction as the adaptation direction and showed a recovery from

342 adaptation in relation to the angular difference between adaptation and test direction

343 (Fabbri et al. 2010). Here we investigated whether directionally-tuned neuronal

344 populations are sensitive to movement amplitude. In particular, we were interested in 
345 measuring the sensitivity for movement amplitude and direction in M1 and PMd, as

346 reported in monkeys, and in other directionally-tuned regions, especially in parietal areas.

347 Results from frontal regions in the left hemisphere are shown in Figure 3a, separately for

348 test trials following adaptation to small (upper row) and large (lower row) amplitude

349 movements as a function of test amplitude (red: small, blue: large) and angular difference

350 between adaptation and test direction. When movement amplitude was kept constant

351 between adaptation and test trials (adapt small, test small; adapt large, test large), we

352 observed the strongest adaptation for the adapted movement direction (angular

353 difference: $0^{\circ}$ ), with a rebound of the BOLD signal for angular difference of $+/-45^{\circ}$, and,

354 to some extent, for angular differences of $+/-90^{\circ}$, in line with our previous findings

355 (Fabbri et al. 2010). When participants were adapted to the small amplitude and tested

356 with the large amplitude (upper row, blue curve), tuning curves were essentially flat,

357 indicating no transfer from the adapted to the non-adapted amplitude. When participants

358 were adapted to large amplitude movements and tested with small amplitude movements

359 (lower row, red curve), there were clear signs of directional tuning, indicating a transfer

360 of adaptation from the adapted to the non-adapted amplitude.

361 Results from parietal regions in the left hemisphere are shown in Figure 3b. Similar to 362 the results in frontal regions, parietal areas showed clear signs of directional tuning when 363 adaptation and test amplitudes were kept constant (upper row: adapt small, test small, red 364 curve; lower row: adapt large, test large, blue curve): the BOLD signal was maximally 365 adapted for movements in the same direction as the adaptation direction $\left(0^{\circ}\right.$ angular 20 
366 difference) and showed a recovery from adaptation in relation to the angular difference

367 between adaptation and test direction. By contrast, there is almost no transfer from the

368 adapted to the non-adapted amplitude when adaptation and test amplitudes differed, as

369 indicated by essentially flat tuning curves (upper row: adapt small, test large, blue curve;

370 lower row: adapt large, test small, red curve).

$371<<$ Add Figure 3 here $>>$

372 The results of the corresponding ROIs in the right hemisphere are shown in Figure 4.

373 Results in the right hemisphere essentially reproduce the same pattern observed in the left

374 hemisphere.

$375<<$ Add Figure 4 here $>>$

376 To quantify our observations we carried out a repeated-measures ANOVA on the beta

377 estimates with the factors Adaptation Amplitude (small, large), Test Amplitude (small,

378 large) and Angular Difference between Adaptation and Test Direction (abbreviated as

379 "Direction" in the following section), separately for each ROI. This analysis revealed a

380 significant interaction between Adaptation Amplitude, Test Amplitude and Direction in

381 each single ROI of the left hemisphere (see results in the section Full Model in Table 2).

382 Based on Figure 3a, we reasoned that the nature of this 3-way interaction in frontal areas

383 was due to a significant 2-way interaction for the factors Test Amplitude and Direction

384 for small adaptation amplitudes (Figure 3a, upper row), and a non-significant 2-way

385 interaction for the factor Test Amplitude and Direction for large adaptation amplitudes 
386 (Figure 3a, lower row). The results of the corresponding statistics supported our

387 observations in each of the areas shown in Figure 3a (see results of frontal regions in 388 sections Adapt Small and Adapt Large in Table 2). Next, we reasoned that parietal areas

389 (Figure 3b) should show a significant 2-way interaction for the factors Test Amplitude 390 and Direction both for small (Figure 3b, upper row) and for large (Figure 3b, lower row)

391 adaptation amplitudes. The corresponding statistics confirmed these predictions in all

392 areas shown in Figure 3b (see results of parietal regions in sections Adapt Small and

393 Adapt Large in Table 2), with the exception of pIPS LH, where the 2-way interaction 394 was significant for large, but not for small adaptation amplitudes. Statistical analysis 395 confirmed a similar pattern in the right hemisphere (Table 3).

$396<<$ Add Table 2 here $>>$

$397<<$ Add Table 3 here $>>$

398 Finally, to quantify which areas showed directional tuning, we carried out a complex

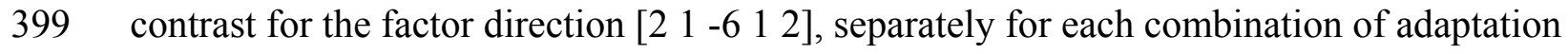
400 amplitude and test amplitude. We reasoned that areas that show directional tuning should 401 show a significant effect for this contrast when adaptation and test amplitude are identical 402 (adapt small, test small; adapt large, test large). Furthermore we reasoned that those areas 403 showing a transfer of adaptation from large amplitude to small amplitude test movements 404 should also show a significant effect for this contrast. The results of these complex 405 contrasts are visualized in Figures $\mathbf{3}$ and $\mathbf{4}$ (significance level indicated by small asterisks 406 next to the corresponding curve). Both frontal and parietal areas showed significant 22 
407 directional tuning as revealed by the complex contrast when adaptation and test

408 amplitudes were identical. In frontal areas, directional tuning was also present when

409 participants were adapted to the large amplitude and tested with the small amplitude, but

410 not vice versa. None of the parietal areas showed significant directional tuning when

411 adaptation and test amplitudes differed, except for left SPOC and right aPCu. Note,

412 however, that in contrast to frontal areas, these two regions showed a significant

413 interaction for the factors Test Amplitude and Direction, indicating that the transfer from

414 the adapted to the non-adapted amplitude was weak and qualitatively different from the 415 transfer observed in frontal areas.

\section{$416 \quad$ Kinematics}

417 Participants performed the task accurately, as indicated by Figure 5, which shows the

418 2D-trajectories of the hand reaching movements of one representative participant during

419 small (Figure 5a) and large (Figure 5b) amplitude movements. The target movement

420 direction is color-coded. A certain amount of variation around the target location is

421 expected due to the fact that there was no visual feedback. However, as can be seen, both

422 direction and amplitude were chosen correctly in almost every trial.

$423<<$ add figure 5 here $>>$

424 Figure 6 shows that spatial accuracy differed between conditions, both along the 425 horizontal (Figure 6a) and vertical (Figure 6b) dimension. In particular, horizontal 426 errors were smaller during small than large amplitude movements and during $0^{\circ}$ 
427 compared to other angular differences. Vertical errors were bigger during movements 428 executed after small than large adaptation amplitudes.

$429<<$ add figure 6 here $>>$

430 For statistical analysis, we submitted the standard deviation of the horizontal and vertical 431 variable error to a 2 (Adaptation Amplitude) x 2 (Test Amplitude) x 5 (Direction) 432 repeated-measures ANOVA. Mean horizontal variable error was $1.38 \mathrm{~cm}$. The horizontal 433 variable error was larger for large in comparison to small amplitude test movements $[\mathrm{F}(1$, 434 4) $=12.428, \mathrm{p}=.024$; small amplitude: $1.1 \mathrm{~cm}$, large amplitude: $1.7 \mathrm{~cm}]$. There was a 435 marginally significant main effect of adaptation amplitude $[\mathrm{F}(1,4)=6.328, \mathrm{p}=.066$;

436 small amplitude: $1.96 \mathrm{~cm}$; large amplitude: $0.81 \mathrm{~cm}$ ]. The horizontal variable error varied 437 with movement direction $\left[\mathrm{F}(4,16)=5.254, \mathrm{p}=.007 ;-90^{\circ} /-45^{\circ} / 0^{\circ} /+45^{\circ} /+90^{\circ}: 1.9 / 1.2 /\right.$ $4380.5 / 1.5 / 1.8 \mathrm{~cm}]$, and was modulated by the interaction between adaptation amplitude 439 and movement direction $[F(4,16)=4.829, \mathrm{p}=.01]$. All remaining interactions and main 440 effects were non-significant (all $\mathrm{p}>.1$ ). Mean vertical variable error was $1.05 \mathrm{~cm}$. 441 Vertical variable error was larger when participants were adapted to small $(1.4 \mathrm{~cm})$ in 442 comparison to large $(0.6 \mathrm{~cm})$ amplitude movements $[\mathrm{F}(1,4)=31.426, \mathrm{p}=.005]$. Vertical 443 variable error was modulated by the interaction between adaptation amplitude and 444 direction $[F(4,16)=5.358, p=.006]$. No other interaction was significant (all $\mathrm{p}>.2)$.

445 Finally, we tested whether movement velocity covaried with movement amplitude and 446 direction. Figure 7 shows that velocity varied with movement amplitude but not with 
447 movement direction: small movements were faster than large movements, similarly for 448 all directions.

$449<<$ add figure 7 here $>>$

450 We submitted the peak velocity to a 2 (Adaptation Amplitude) x 2 (Test Amplitude) x 5

451 (Movement Direction) repeated-measures ANOVA. Across conditions, mean peak 452 velocity was $38.96 \mathrm{~cm} / \mathrm{s}$. Large amplitude test trials were faster $(45.96 \mathrm{~cm} / \mathrm{s})$ than small 453 amplitude test trials $(31.95 \mathrm{~cm} / \mathrm{s})[\mathrm{F}(1,4)=45.51, \mathrm{p}=.003]$. Likewise, movements were 454 faster when participants were adapted to large $(40.05 \mathrm{~cm} / \mathrm{s})$ in comparison to small $455(37.86 \mathrm{~cm} / \mathrm{s})$ amplitude movements $[\mathrm{F}(1,4)=8.029, \mathrm{p}=.049]$. Peak velocity did not 456 vary with direction $[\mathrm{F}(4,16)=1.591, \mathrm{p}=.225]$, and none of the interactions were 457 significant (all $\mathrm{p}>.16)$. 


\section{Discussion}

\section{Sensitivity for movement amplitude in directionally-tuned neuronal populations}

461 Using fMRI adaptation, we recently reported evidence for directionally-tuned neurons in

462 several areas of the human visuomotor system (Fabbri et al. 2010). Here we investigated

463 the extent to which directionally-tuned neuronal populations are sensitive to movement

464 amplitude. To this aim, we adapted participants to the execution of reaching movements

465 in one specific direction and amplitude, and measured the recovery from adaptation of the

466 BOLD signal during the execution of movements at varying directions with either the

467 adapted or non-adapted amplitude.

468 When movement amplitude was kept constant between adaptation and test trials, the

469 BOLD signal in bilateral Insula, PMv, SMA, PMd, left M1, bilateral IPL, aIPS, pIPS,

470 SPOC and right aPCu showed clear signs of directional selectivity: adaptation was

471 strongest during test trials with $0^{\circ}$ angular difference from the adaptation direction and

472 decreased as the angular difference increased, in line with our previous results (Fabbri et

473 al. 2010). This pattern of directional selectivity was similar for small $(6 \mathrm{~cm})$ and large (12

$474 \mathrm{~cm})$ amplitude movements.

475 When movement amplitude was varied between adaptation and test trials, parietal areas

476 showed no transfer from the adapted to the non-adapted amplitude. This observation was

477 the same for adaptation to small and adaptation to large amplitude movements. By

478 contrast, we observed a partial transfer from the adapted to the non-adapted amplitude

479 when participants were adapted to the large and tested with the small amplitude in frontal 26 
480 areas. We observed no such transfer when participants were adapted to the small 481 amplitude and tested with large amplitude movements.

482 Messier and Kalaska (2000) reported that a sizeable number of PMd cells showed a main 483 effect of direction alone in different behavioral epochs of a trial, in an instructed-delay 484 task in which prior information about direction and amplitude had to be memorized 485 before movement initiation. In particular, the percentage of cells sensitive to direction 486 only decreased from $59 \%$ in the epoch when the information was given, to $35 \%$ in the 487 epoch of movement execution. In contrast, very few cells (2-4\%) showed a main effect of 488 only movement amplitude in a given epoch. Our paradigm does not allow distinguishing 489 between different epochs of a trial, so we cannot rule out that there exist neuronal 490 populations that code only movement direction during the movement planning phase, and 491 not during the movement execution phase. However, if the importance of movement 492 amplitude were to increase over the course of planning and executing a movement, one 493 might assume that the representation of movement amplitude would differ between 494 parietal areas, which are mainly involved with the planning of the movement (Andersen 495 and Buneo 2002; Andersen et al. 1997; Kalaska et al. 1997), and frontal areas, which are 496 closer to the execution of the movement. In line with this view, our results suggest 497 different organizing principles between frontal and parietal regions, as will be discussed 498 in the following sections. 
502 We observed that directionally-tuned parietal regions show no transfer from the adapted

503 to the non-adapted amplitude, suggesting that these areas are sensitive to specific

504 combinations of movement direction and amplitude. Several authors have argued for a

505 posterior-to-anterior gradient in parietal cortex, with more posterior regions such as pIPS

506 and SPOC more sensitive to visual aspects of reaching, grasping or the visual location of

507 targets, and anterior regions such as mIPS and aPCu involved in the encoding of motor

508 aspects of actions such as proprioception (e.g. Filimon et al. 2009; Stark and Zohary

509 2008). In line with this view, we recently demonstrated that the modulation of directional

510 tuning by the type of motor act increases from posterior to anterior regions (Fabbri et al.

511 2010). A similar gradient is likely to underlie the representation of movement direction

512 and amplitude, with posterior regions encoding the spatial location of the endpoint of the

513 movement, and anterior regions involved in the encoding of the displacement of effectors

514 from the start to the goal position in spatial coordinates.

\section{The representation of movement direction and amplitude in frontal areas}

516 In contrast to parietal regions, frontal regions showed a partial transfer of adaptation to

517 movement direction from the large to the small amplitude, but not vice versa. Several

518 mechanisms might underlie this finding:

519 i. Let us assume that one of the organizing principles in frontal regions is an 520 arrangement in motor maps coding both movement direction and amplitude, 521 similar to the motor maps described in the superior colliculus (Lee et al. 1988) 
(see Figure 8a). If participants are adapted to large amplitude movements, it is possible that the entire range of amplitudes from the start to the end position would be activated (Figure 8a, left column). As a result, when participants are tested with a small amplitude movement, we should expect to see transfer since this amplitude was adapted during large amplitude movements. By contrast, no such transfer should occur from the small to the large amplitude movement

ii. Alternatively, we might assume that the size of motor fields representing the end points of movements on these motor maps increase with increasing movement amplitude (see Figure 8b), in line with the fact that the precision of reaching movements tend to decrease with movement amplitude. If that were the case, adaptation to small amplitudes would be expected to lead to relatively small transfer to large amplitudes (Figure 8b, left column). By contrast, adaptation to large amplitudes would be expected to lead to larger amounts of transfer when

538 iii. Finally, movement amplitude in frontal, but not in parietal areas, might be coded 539 by discharge rate, with increasing amplitudes leading to larger discharge rates (Figure 8 c, upper row). Such an explanation would be in line with neurophysiological studies that reported an increase in firing rates in frontal areas with increasing movement amplitude (Fu et al. 1995; Fu et al. 1993). We assume that adaptation is proportional to the initial strength of activation (Figure 8 c, 
middle row). Consequently, when participants are adapted to large amplitude

545 movements and tested with small amplitude movements, tuning curves would be

546 expected to be more pronounced in comparison to adaptation to small amplitude

547 movements followed by adaptation to large amplitude movements (Figure 8 c,

$548 \quad$ lower row).

$549<<$ add figure 8 here $>>$

\section{Serial correlations in fMRI data}

551 fMRI time courses are correlated from one scan (or time point) to the next (see also

552 Christova et al. 2011). It is therefore not correct to assume that the degrees of freedom

553 (df) are simply the number of scans minus the dfs used in the model; there should be

554 fewer. This inflates the p-values in fixed-effects models (i.e., when model parameters are

555 estimated based on the concatenated data of all participants), and a correction for

556 autocorrelation would be due for statistical inferences pertaining to the group.

557 However, in order to make population-inferences (as opposed to the group of 558 participants) we performed a hierarchical random effects analysis in which a summary

559 statistic for each subject and voxel was calculated (in our case a beta estimate) (see e.g.

560 Holmes and Friston 1998; Penny et al. 2003). These summary statistics were then

561 submitted to a random effects analysis, looking at the variance between effect sizes as a

562 random effect ( $\mathrm{df}=$ number of subjects -1$)$. Accordingly, the p-values of the fixed-

563 effects analysis, which might be inflated because of serial correlations, did not enter this

564 second-level analysis since the critical standard error was based on the difference of 30 
565 parameter estimates between subjects. As a consequence, any wrong assumption of the p-

566 values of the fixed effects analysis does not affect the results of our random-effects 567 analysis.

568 Finally, we compared the statistical maps revealed by the contrast "test trials adapted

569 direction, adapted amplitude versus all remaining test trials" with and without correction

570 for serial correlation. Correction for serial correlations was performed within

571 BrainVoyager by computing a RFX GLM without correcting for serial correlations,

572 computing the one-lag autocorrelation $[\mathrm{AR}(1)]$ of the residuals, subtracting these from

573 each time course, and recomputing the RFX GLM on the basis of these adjusted time

574 courses. Both the corrected and the non-corrected RFX GLM revealed the same regions,

575 with a slightly wider extent for the corrected in comparison for the non-corrected RFX

576 GLM. In summary, this shows that our results were stable and unbiased.

\section{Possible complicating factors}

578 It is known that movement amplitude co-varies with velocity and that neurons in M1 are

579 sensitive to movement velocity (Moran and Schwartz 1999). Indeed, we measured an

580 increase in movement velocity with increasing movement amplitude in our kinematics

581 experiment. Does this mean that our experiment measured sensitivity to movement speed

582 rather than movement amplitude? If this were the case, we would have expected to have 583 seen an interaction between adaptation and test amplitude, as well as an interaction

584 between adaptation amplitude, test amplitude and movement direction in the kinematic 
585 data. We observed none of these effects. Therefore, differences in movement speed

586 between small and large amplitude movements alone cannot explain our results.

587 How would a covariation between movement amplitude and speed affect the 588 interpretation of our results? We observed that parietal areas showed no transfer of 589 adaptation to movement direction from the adapted to the non-adapted amplitude, 590 whereas frontal areas showed a partial transfer from large to small amplitudes, but not 591 vice versa. This finding holds irrespective of whether the difference between the adapted 592 and the non-adapted amplitude was introduced by a change in movement amplitude 593 alone, or whether it was introduced by a change in movement amplitude and speed. 594 Further experiments will be required to quantify the sensitivity to amplitude and speed in 595 directionally tuned neuronal populations.

596 Although we manipulated the visual appearance of the instruction arrows to reduce 597 adaptation for low-level visual features, it could be argued that the direction of the visual 598 instruction alone caused directional tuning. Moreover, direction of the arrow was 599 spatially congruent with the direction of the reaching movement, while the color of the 600 arrow was arbitrarily associated to the amplitude of the reaching movement. To test 601 whether the modality of the instruction could account for our results, we conducted a 602 control experiment (not reported here) where we instructed participants about the 603 direction of the movement following the procedure used in our previous study (Fabbri et 604 al. 2010) but using arbitrary auditory cues (e.g. "A" indicated the central left target). The 605 control experiment identified directional tuning in similar regions as those reported in the 32 
606 current study, indicating that the results are due to the direction of the reaching 607 movement and not to the visual instructions.

\section{Conclusions}

609 Our results show that sensitivity for movement direction and amplitude is not restricted to

610 PMd and M1, but extends to PMv, SMA, the insula as well as several anterior and

611 posterior parietal regions. Our results suggest that parietal areas represent specific 612 combinations of movement amplitude and direction, while frontal areas show some 613 transfer from the adapted to the non-adapted amplitude. These results provide an 614 important extension of our knowledge about the representation of reaching movements. 


\section{Acknowledgements}

616 We are grateful to Jens Schwarzbach for many helpful discussions and to Corrado

617 Caudek for comments on the manuscript. We would also like to thank Dr. Maxim Zaitsev

618 of the University Hospital Freiburg for providing the Point-Spread Function and modified

619 EPI sequences. 


\section{Grants}

621 This research was supported by the Provincia Autonoma di Trento and the Fondazione

622 Cassa di Risparmio di Trento e Rovereto.

623 


\section{Disclosures}

625 No conflicts of interest, financial or otherwise, are declared by the author(s). 
627 Andersen RA, and Buneo CA. Intentional maps in posterior parietal cortex. Annual review 628 of neuroscience 25: 189-220, 2002.

629 Andersen RA, Snyder LH, Bradley DC, and Xing J. Multimodal representation of space in 630 the posterior parietal cortex and its use in planning movements. Annual review of 631 neuroscience 20: 303-330, 1997.

632 Beurze SM, de Lange FP, Toni I, and Medendorp WP. Spatial and effector processing in 633 the human parietofrontal network for reaches and saccades. Journal of neurophysiology 101: 634 3053-3062, 2009.

635 Brainard DH. The Psychophysics Toolbox. Spatial vision 10: 433-436, 1997.

636 Brouwer AM, Brenner E, and Smeets JB. Hitting moving objects. The dependency of hand 637 velocity on the speed of the target. Exp Brain Res 133: 242-248, 2000.

638 Caminiti R, Johnson PB, Galli C, Ferraina S, and Burnod Y. Making arm movements 639 within different parts of space: the premotor and motor cortical representation of a 640 coordinate system for reaching to visual targets. J Neurosci 11: 1182-1197, 1991.

641 Cavina-Pratesi C, Monaco S, Fattori P, Galletti C, McAdam TD, Quinlan DJ, Goodale MA, 642 and Culham JC. Functional magnetic resonance imaging reveals the neural substrates of 643 arm transport and grip formation in reach-to-grasp actions in humans. J Neurosci 30: 644 10306-10323, 2010.

645 Christova P, Lewis SM, Jerde TA, Lynch JK, and Georgopoulos AP. True associations 646 between resting fMRI time series based on innovations. J Neural Eng 8: 046025, 2011.

647 Connolly JD, Goodale MA, Cant JS, and Munoz DP. Effector-specific fields for motor 648 preparation in the human frontal cortex. NeuroImage 34: 1209-1219, 2007.

649 Culham JC, Danckert SL, DeSouza JF, Gati JS, Menon RS, and Goodale MA. Visually guided grasping produces fMRI activation in dorsal but not ventral stream brain areas. Experimental brain research Experimentelle Hirnforschung 153: 180-189, 2003.

Desmurget M, Grea H, Grethe JS, Prablanc C, Alexander GE, and Grafton ST. Functional anatomy of nonvisual feedback loops during reaching: a positron emission tomography study. J Neurosci 21: 2919-2928, 2001.

Eisenberg M, Shmuelof L, Vaadia E, and Zohary E. Functional organization of human motor cortex: directional selectivity for movement. J Neurosci 30: 8897-8905, 2010.

Fabbri S, Caramazza A, and Lingnau A. Tuning curves for movement direction in the human visuomotor system. J Neurosci 30: 13488-13498, 2010.

Fernandez-Ruiz J, Goltz HC, DeSouza JF, Vilis T, and Crawford JD. Human parietal "reach region" primarily encodes intrinsic visual direction, not extrinsic movement direction, in a visual motor dissociation task. Cereb Cortex 17: 2283-2292, 2007.

Filimon F, Nelson JD, Hagler DJ, and Sereno MI. Human cortical representations for reaching: mirror neurons for execution, observation, and imagery. NeuroImage 37: 13151328, 2007.

Filimon F, Nelson JD, Huang RS, and Sereno MI. Multiple parietal reach regions in humans: cortical representations for visual and proprioceptive feedback during on-line reaching. J Neurosci 29: 2961-2971, 2009.

Fortier PA, Kalaska JF, and Smith AM. Cerebellar neuronal activity related to whole-arm reaching movements in the monkey. Journal of neurophysiology 62: 198-211, 1989. 
670 Frey SH, Vinton D, Norlund R, and Grafton ST. Cortical topography of human anterior 671 intraparietal cortex active during visually guided grasping. Brain Res Cogn Brain Res 23: 672 397-405, 2005.

673 Friston KJ, Fletcher P, Josephs O, Holmes A, Rugg MD, and Turner R. Event-related 674 fMRI: characterizing differential responses. NeuroImage 7: 30-40, 1998.

675 Fu QG, Flament D, Coltz JD, and Ebner TJ. Temporal encoding of movement kinematics in 676 the discharge of primate primary motor and premotor neurons. Journal of neurophysiology 677 73: 836-854, 1995.

678 Fu QG, Suarez JI, and Ebner TJ. Neuronal specification of direction and distance during 679 reaching movements in the superior precentral premotor area and primary motor cortex of 680 monkeys. Journal of neurophysiology 70: 2097-2116, 1993.

681 Georgopoulos AP, Kalaska JF, Caminiti R, and Massey JT. On the relations between the 682 direction of two-dimensional arm movements and cell discharge in primate motor cortex. $J$ 683 Neurosci 2: 1527-1537, 1982.

684 Grill-Spector $\mathbf{K}$, and Malach R. fMR-adaptation: a tool for studying the functional 685 properties of human cortical neurons. Acta psychologica 107: 293-321, 2001.

686 Haxby JV, Gobbini MI, Furey ML, Ishai A, Schouten JL, and Pietrini P. Distributed and 687 overlapping representations of faces and objects in ventral temporal cortex. Science (New $688 \quad$ York, NY 293: 2425-2430, 2001.

689 Holmes AP, and Friston KJ. Generalisability, random effects and population inference. $690 \quad$ NeuroImage 7: S754, 1998.

691 Kakei S, Hoffman DS, and Strick PL. Direction of action is represented in the ventral premotor cortex. Nature neuroscience 4: 1020-1025, 2001.

Kalaska JF, Caminiti R, and Georgopoulos AP. Cortical mechanisms related to the direction of two-dimensional arm movements: relations in parietal area 5 and comparison with motor cortex. Experimental brain research 51: 247-260, 1983.

Kalaska JF, Scott SH, Cisek P, and Sergio LE. Cortical control of reaching movements. Current opinion in neurobiology 7: 849-859, 1997.

Kriegeskorte N, Simmons WK, Bellgowan PS, and Baker CI. Circular analysis in systems neuroscience: the dangers of double dipping. Nature neuroscience 12: 535-540, 2009.

Kurata K. Premotor cortex of monkeys: set- and movement-related activity reflecting amplitude and direction of wrist movements. Journal of neurophysiology 69: 187-200, 1993.

Lee C, Rohrer WH, and Sparks DL. Population coding of saccadic eye movements by neurons in the superior colliculus. Nature 332: 357-360, 1988.

Messier J, and Kalaska JF. Covariation of primate dorsal premotor cell activity with direction and amplitude during a memorized-delay reaching task. Journal of neurophysiology 84: 152-165, 2000.

Moran DW, and Schwartz AB. Motor cortical representation of speed and direction during reaching. Journal of neurophysiology 82: 2676-2692, 1999.

Pelli DG. The VideoToolbox software for visual psychophysics: transforming numbers into movies. Spatial vision 10: 437-442, 1997.

Penny WD, Holmes AP, and Friston KJ. Random effects analysis. In: Human Brain Function, edited by Frackowiak RSJ, Ashburner J, Penny WD, Zeki S, Friston KJ, Frith CD, Dolan RJ, and Price CJElsevier, 2003. 
714 Riehle A, and Requin J. Monkey primary motor and premotor cortex: single-cell activity 715 related to prior information about direction and extent of an intended movement. Journal of 716 neurophysiology 61: 534-549, 1989.

717 Schwarzbach J. A simple framework (ASF) for behavioral and neuroimaging experiments 718 based on the psychophysics toolbox for MATLAB. Behav Res Methods 2011.

719 Stark A, and Zohary E. Parietal mapping of visuomotor transformations during human tool 720 grasping. Cereb Cortex 18: 2358-2368, 2008.

721 Van Der Werf J, Jensen 0, Fries P, and Medendorp WP. Neuronal synchronization in human posterior parietal cortex during reach planning. J Neurosci 30: 1402-1412, 2010. Van Essen DC. Windows on the brain: the emerging role of atlases and databases in neuroscience. Current opinion in neurobiology 12: 574-579, 2002.

Zaitsev M, Hennig J, and Speck 0. Point spread function mapping with parallel imaging techniques and high acceleration factors: fast, robust, and flexible method for echo-planar imaging distortion correction. Magn Reson Med 52: 1156-1166, 2004.

Zeng $\mathbf{H}$, and Constable RT. Image distortion correction in EPI: comparison of field mapping with point spread function mapping. Magn Reson Med 48: 137-146, 2002. 


\section{Figure legends}

733 Figure 1. Paradigm and predictions. (a) Participants lay in the scanner with their index

734 finger on the starting position of a device attached to their stomach, and executed small

735 and large amplitude reaching movements on the device. (b) On the schematic device is

736 shown the full set of angular differences between adaptation and test directions $\left(0^{\circ},+/-\right.$

$73745^{\circ},+/-90^{\circ}$ ) for small (red) and large (blue) amplitude movements. (c) Visual instructions

738 presented on the screen informed participants about the direction (orientation of the

739 arrow) and amplitude (color of the arrow; red: small amplitude, blue: large amplitude) of

740 the reaching movement. The example shows a sequence consisting of three small

741 amplitude adaptation trials (highlighted by dashed rectangle; adaptation direction: central

742 ahead) followed by a large amplitude test trial (indicated by solid line rectangle) with an

743 angular difference of $45^{\circ}$ between adaptation and test direction. (d) Directionally-tuned

744 regions insensitive to movement amplitude should show a transfer of adaptation from the

745 adapted to the non-adapted amplitude, as measured by adaptation in relation to the

746 angular difference between adaptation and test directions, for both adapted (red line) and

747 non-adapted (blue line) amplitude test trials. (e) Regions sensitive to the specific

748 combination of movement direction and amplitude should show directional tuning only

749 for the adapted amplitude and no transfer to the non-adapted amplitude.

750 Figure 2. Regions more active during a change in either movement direction or

751 amplitude compared to baseline consist of bilateral insula, ventral premotor (PMv),

752 supplementary motor area (SMA), dorsal premotor (PMd), left M1, bilateral inferior 40 
753 parietal lobule (IPL), anterior and posterior intraparietal sulcus (aIPS and pIPS,

754 respectively), right anterior precuneus $(\mathrm{aPCu})$ and superior parieto-occipital cortex 755 (SPOC).

756 Figure 3. fMRI Response (reported as percent signal change) in frontal (a) and parietal

757 (b) regions of the left hemisphere. Results are reported separately for test trials following 758 adaptation to the small (upper row) and the large (lower row) amplitude. Separate lines

759 show the fMRI Response during test trials with small (red) and large (blue) amplitude

760 movements as a function of the angular difference between adaptation and test trial.

761 Asterisks indicate statistical significance of complex contrasts (see Results section for 762 details). *: $\mathrm{p}<.05 ; * *: \mathrm{p}<.01 ; * * *: \mathrm{p}<.0001$

763 Figure 4. fMRI Response (reported as percent signal change) in frontal (a) and parietal

764 (b) regions of the right hemisphere (for labeling conventions see Figure 3).

765 Figure 5. Trajectories of hand reaching movements for small (a) and large (b) amplitude 766 test trials, for one representative participant, collapsed across the entire length of the 767 experiment. Colors indicate target directions (dark blue: $-90^{\circ}$ : light blue: $-45^{\circ}$; green: $0^{\circ}$, 768 yellow: $+45^{\circ}$, red: $90^{\circ}$ ). Gray circles mark the positions of the target locations.

769 Figure 6. Horizontal (a) and vertical (b) error of movements with small (black and dark 770 gray) and large (light gray and white) amplitudes as a function of the angular difference 771 between adaptation and test direction. 
772 Figure 7. Peak velocity $(\mathrm{cm} / \mathrm{s})$ of movements with small and large adaptation and test

773 amplitudes as a function of the angular difference between adaptation and test direction

774 (for labeling conventions see Figure 6).

775 Figure 8. Graphical depiction of three alternative explanations that might underlie the

776 results observed in frontal areas (see text for details). Panels (a) and (b) illustrate two

777 explanations based on movement direction and amplitude organized as motor maps.

778 Panel (c) shows an explanation based on directionally tuned neuronal populations that are

779 up- and down-regulated by movement amplitude. (a, b) Movement direction and

780 amplitude are coded as motor maps similar to those described in the superior colliculus

781 (Lee et al. 1988): amplitude is coded along the horizontal and direction along the vertical

782 dimension (upper row: before adaptation, middle row: during adaptation, lower row:

783 during test trials). (a) We assume that small amplitude movements lead to adaptation for

784 small amplitude movements that gradually decreases with angular difference between the

785 adapted and neighboring directions (a, middle left panel). When adaptation to small

786 amplitudes is followed by testing with large amplitudes, there is no transfer of adaptation

787 since large amplitudes were not adapted (a, lower left panel; strength of adaptation is

788 coded by gray level, darker gray indicates stronger adaptation; length of black arrow

789 indicates amplitude). By contrast, when participants are adapted to large amplitudes, we

790 assume that the entire range of amplitudes, including small ones, are adapted (a, middle

791 right panel). As a result, testing with small amplitudes shows a transfer of adaptation (a,

792 lower right panel). (b) Alternatively, it is possible that the size of motor fields 
793 representing the end points of movements on these motor maps, highlighted by gray

794 discs, increases with movement amplitude. If that were the case, adaptation to small

795 amplitudes (b, middle left panel) would be expected to lead to no or relatively little 796 transfer to the large amplitudes (b, lower left panel; black circle indicates hypothetical

797 size of motor field for tested movement). By contrast, adaptation to large amplitude (b,

798 middle right panel) would be expected to lead to a larger transfer of adaptation when

799 followed by small amplitude movements (b, lower right panel). (c) Explanation based on

800 directionally tuned neuronal populations that are up- and down-regulated by movement

801 amplitude. X-axis represents movement direction, y-axis represents strength of fMRI

802 activation (in arbitrary units) within a hypothetical voxel containing neuronal populations

803 tuned to various different directions. We assume that for small amplitude movements,

804 overall strength of activation is weaker in comparison to large amplitude movements,

805 irrespective of direction (c, upper row). During adaptation, we assume that the strength of

806 activation adapts proportionally to the strength of the signal before adaptation (c, middle

807 row). During large amplitude test trials, we assume that the overall signal increases by a

808 constant factor due to the change from small to large amplitude movements (c, lower left

809 panel). During small amplitude test trials, we assume that the signal decreases by a

810 constant factor due to the change from large to small amplitude movements. The resulting

811 tuning curves are clearly more pronounced for small amplitude test trials (c, lower right 812 panel). 


\section{Table legends}

815 Table 1. Talairach coordinates of regions of interest shown in Figure 2. PMv, ventral

816 premotor cortex; SMA, supplementary motor area; PMd, dorsal premotor cortex; M1,

817 primary motor cortex; IPL, inferior parietal lobule; aIPS, anterior intraparietal sulcus;

$818 \mathrm{aPCu}$, anterior precuneus; pIPS, posterior intraparietal sulcus; SPOC, superior parieto-

819 occipital cortex. LH: left hemisphere, RH: right hemisphere.

820 Table 2. Summary of the statistical analysis of beta estimates as a function of adaptation

821 amplitude, test amplitude and angular difference between adaptation and test direction for

822 ROIs in the left hemisphere. Non-significant effects are highlighted in gray.

823 Table 3. Summary of the statistical analysis of beta estimates as a function of adaptation

824 amplitude, test amplitude and angular difference between adaptation and test direction for

825 ROIs in the right hemisphere. Non-significant effects are highlighted in gray. 

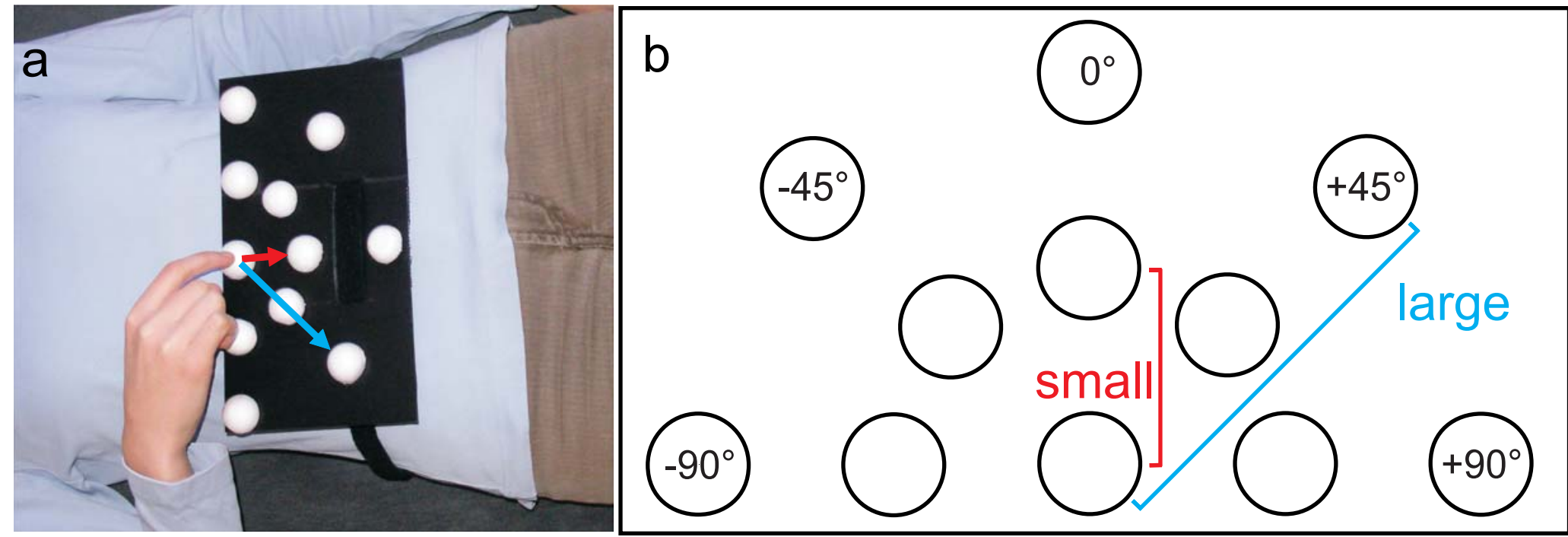

C
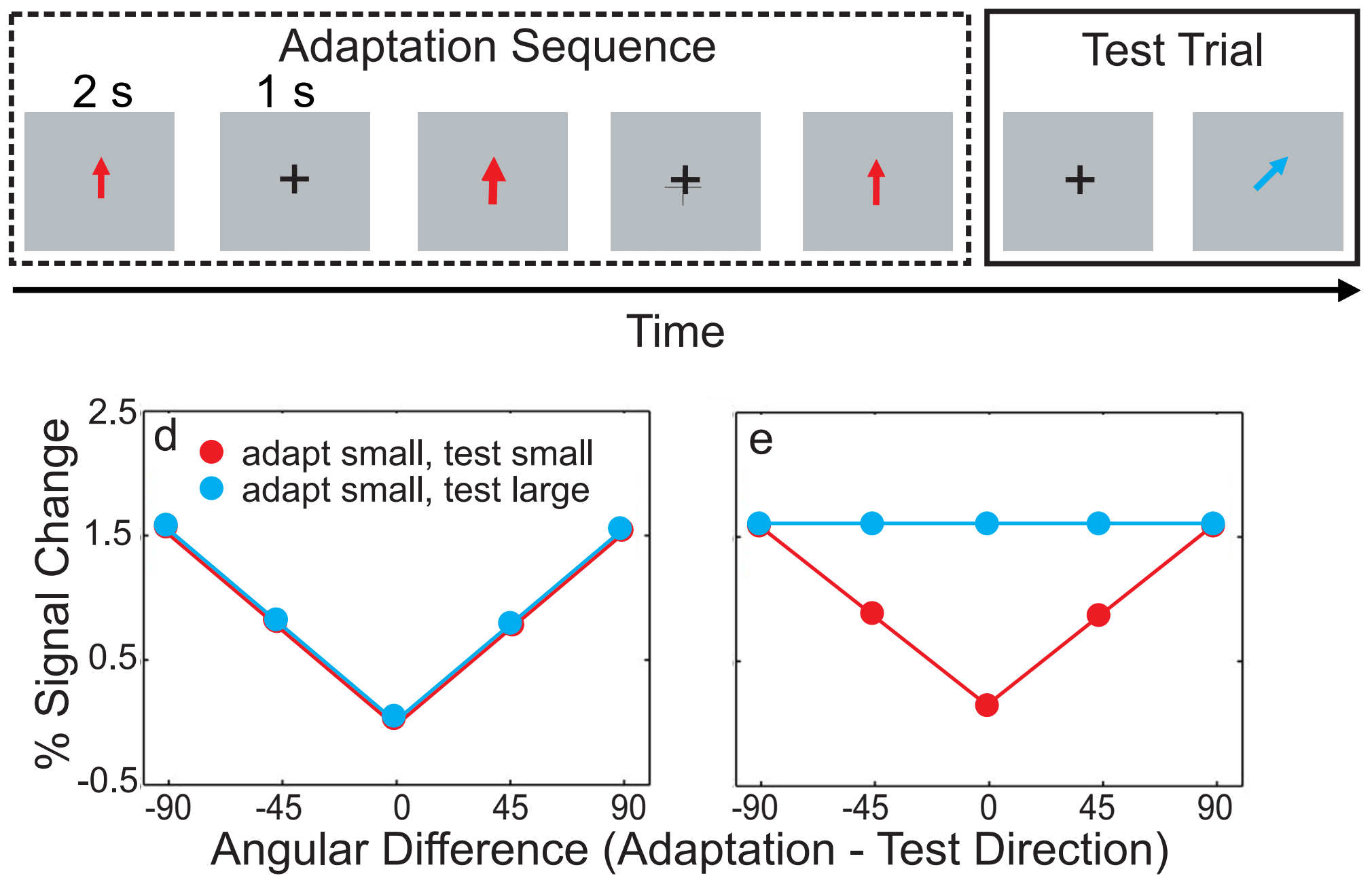


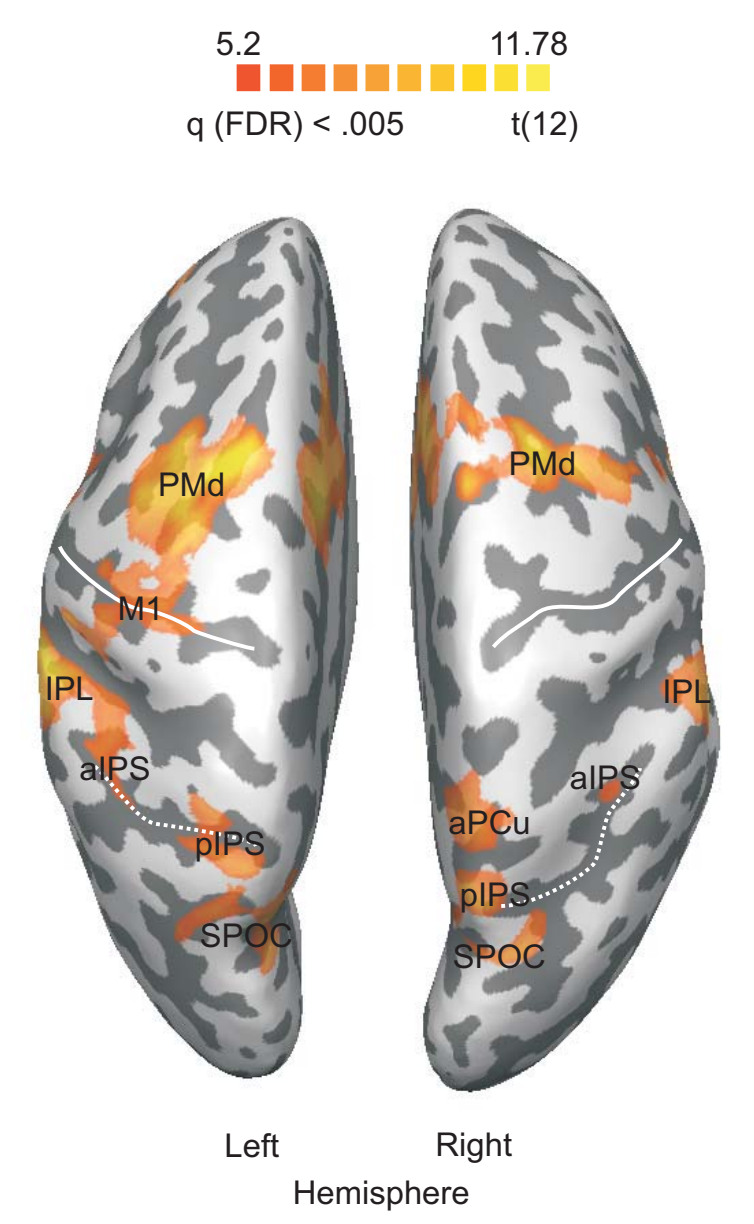

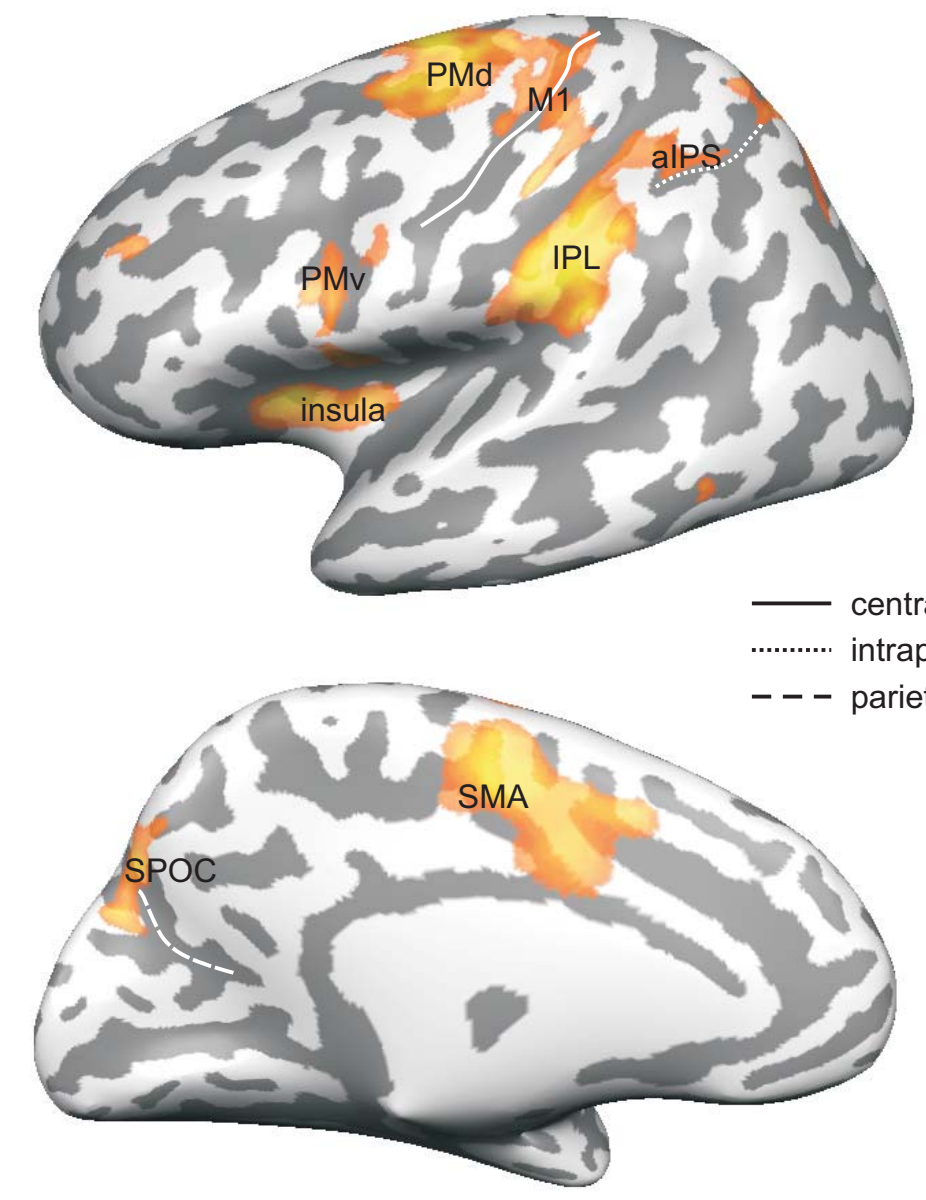

Left Hemisphere
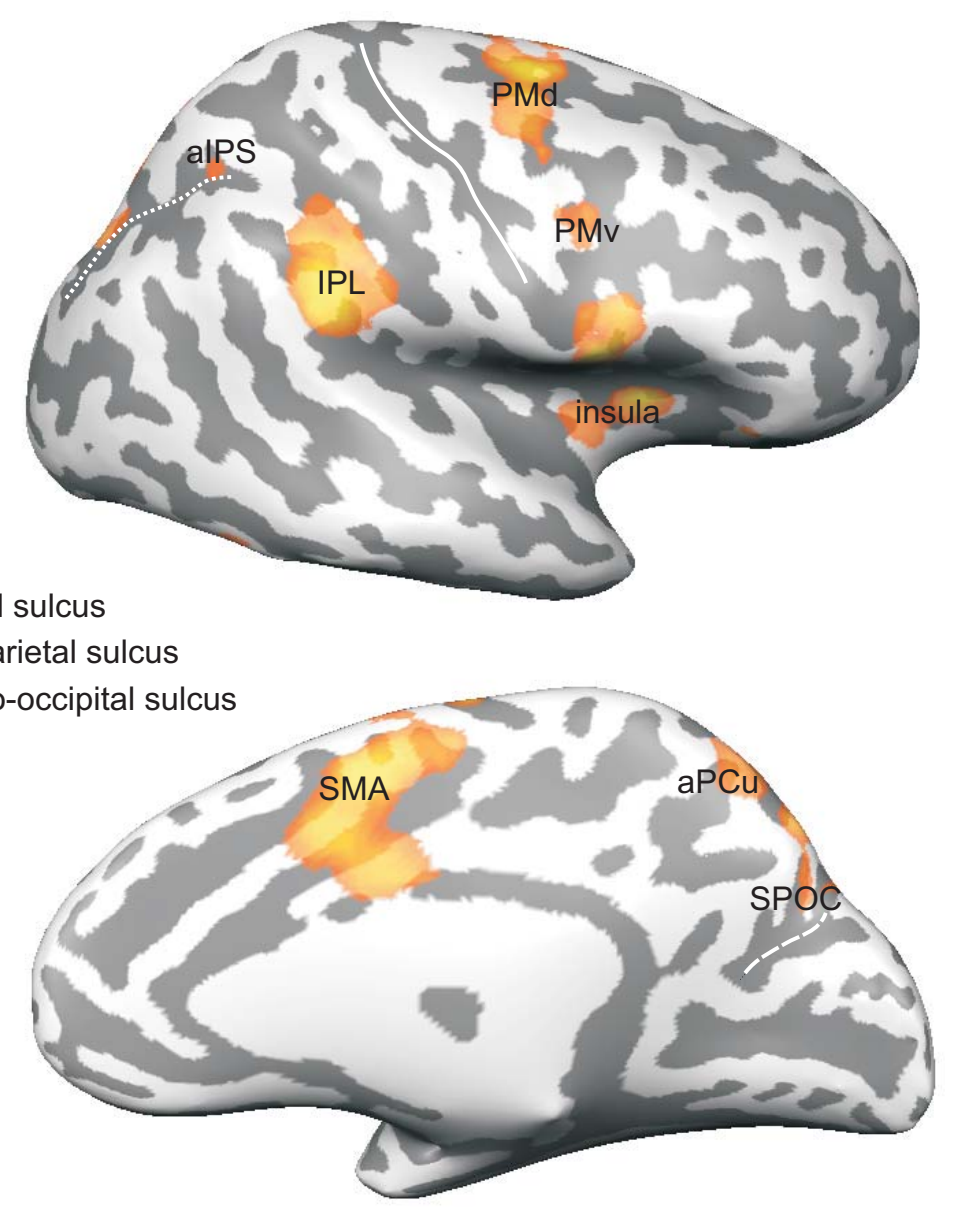

Right Hemisphere 

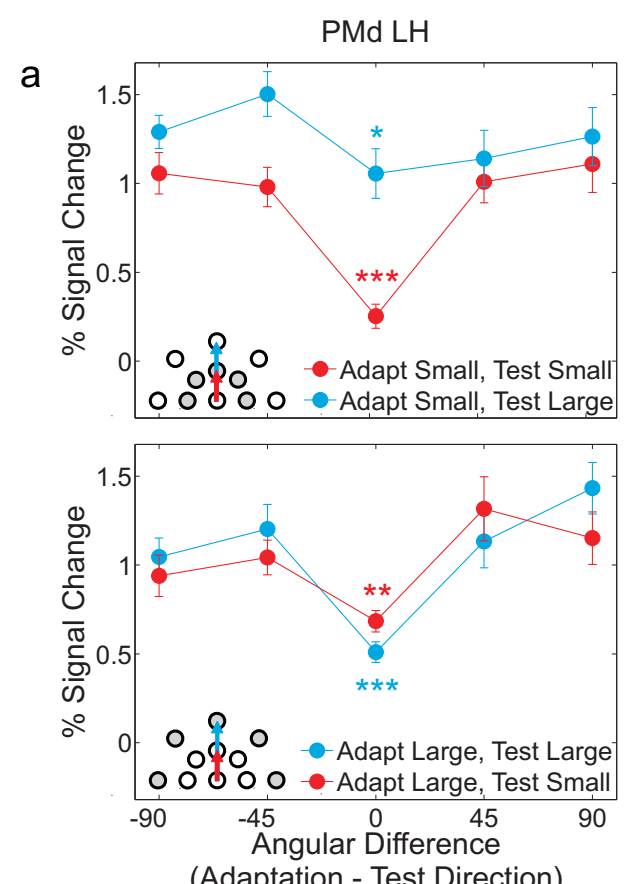

$$
\text { IPL LH }
$$
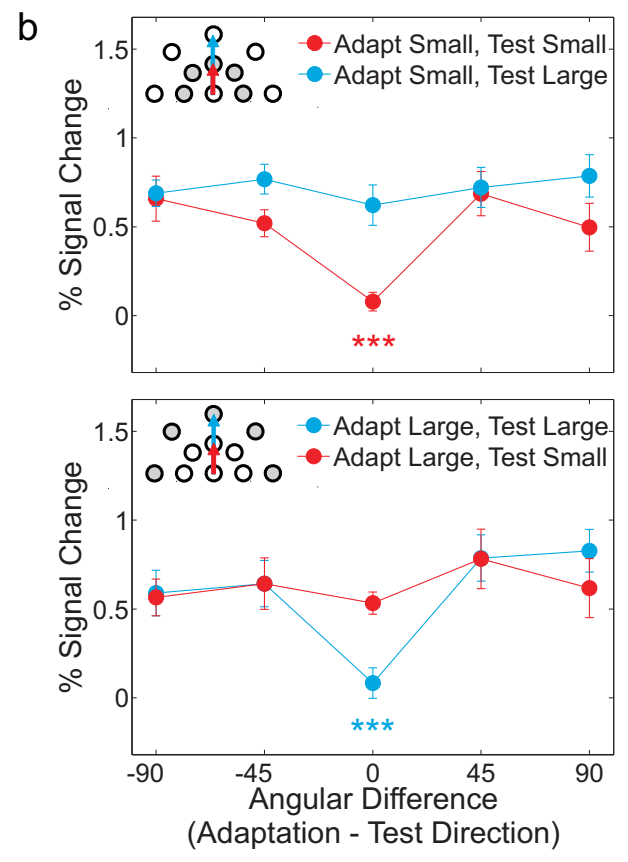
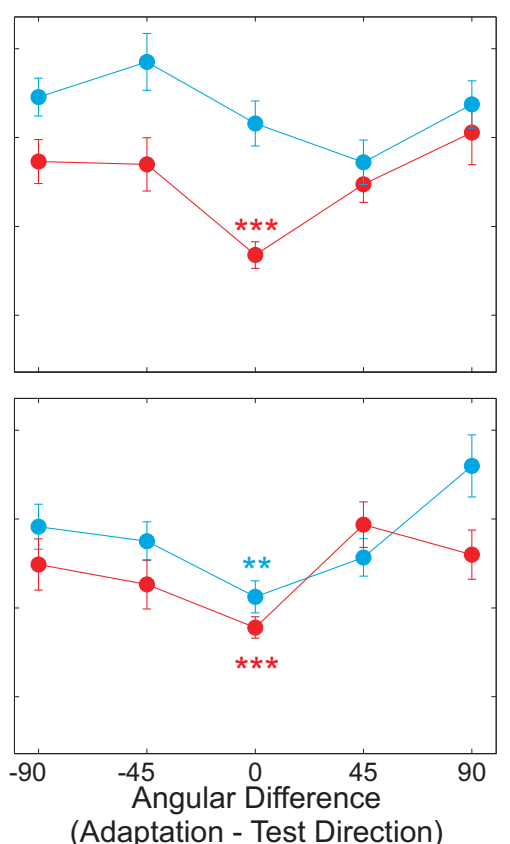

aIPS LH
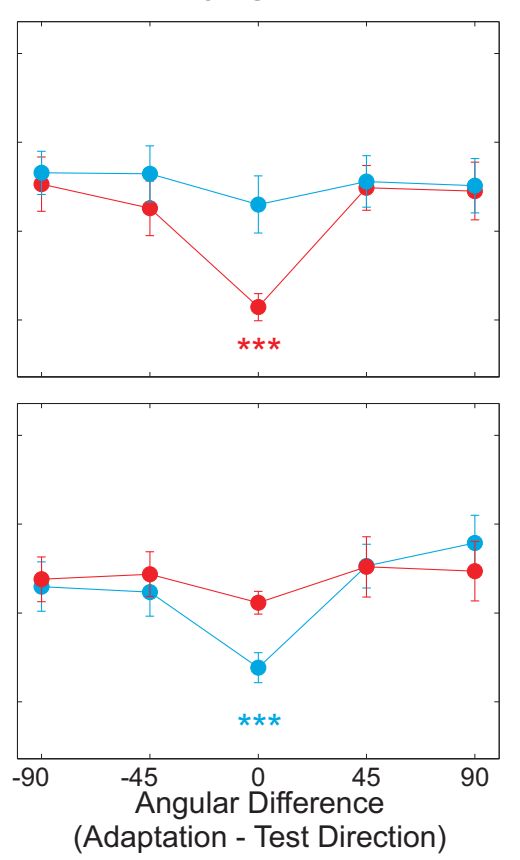

insula LH

SMA LH
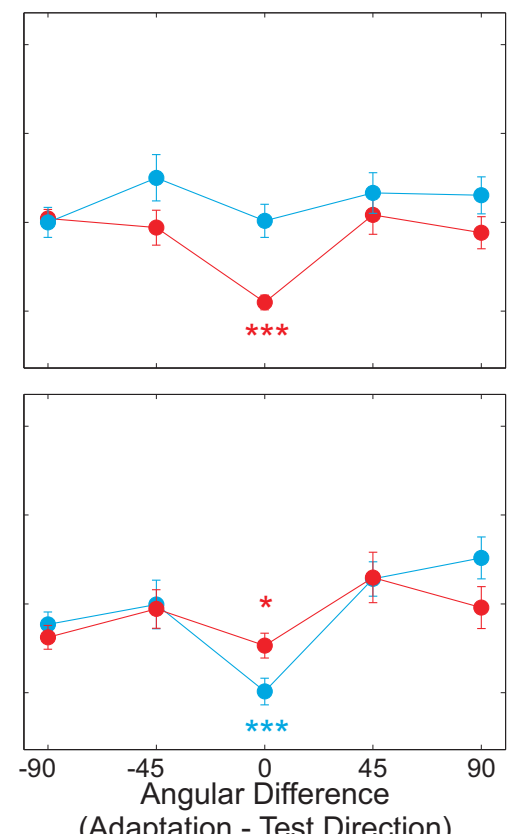

pIPS LH
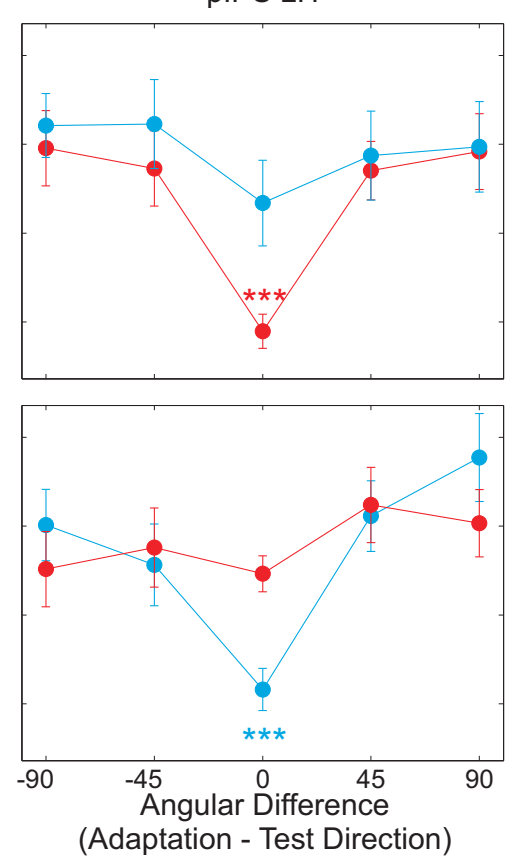

SPOC LH
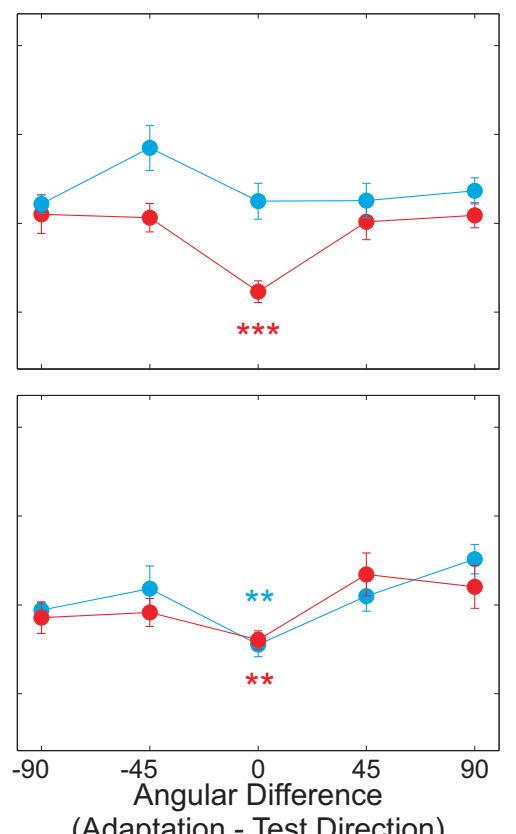
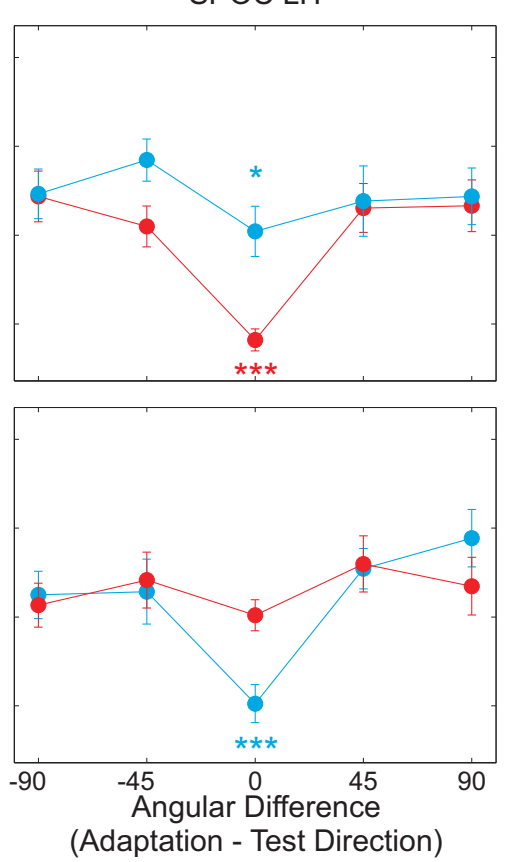

PMv LH
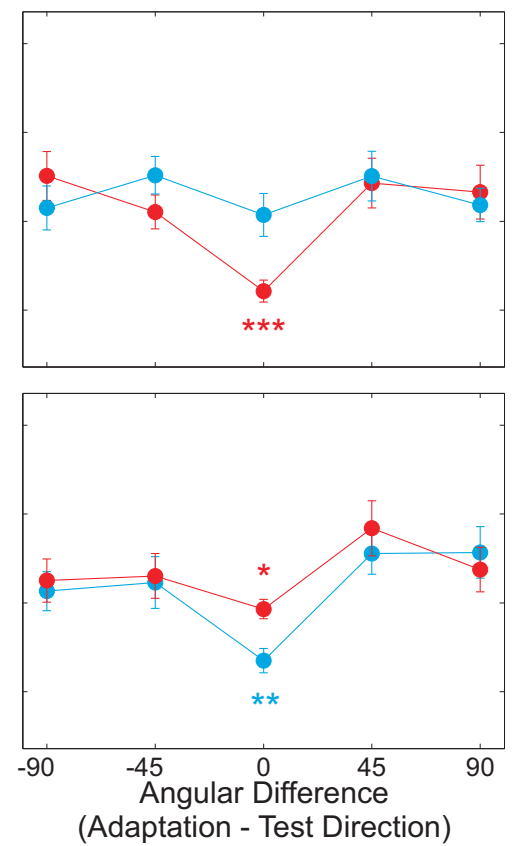

(Adaptation - Test Direction) 

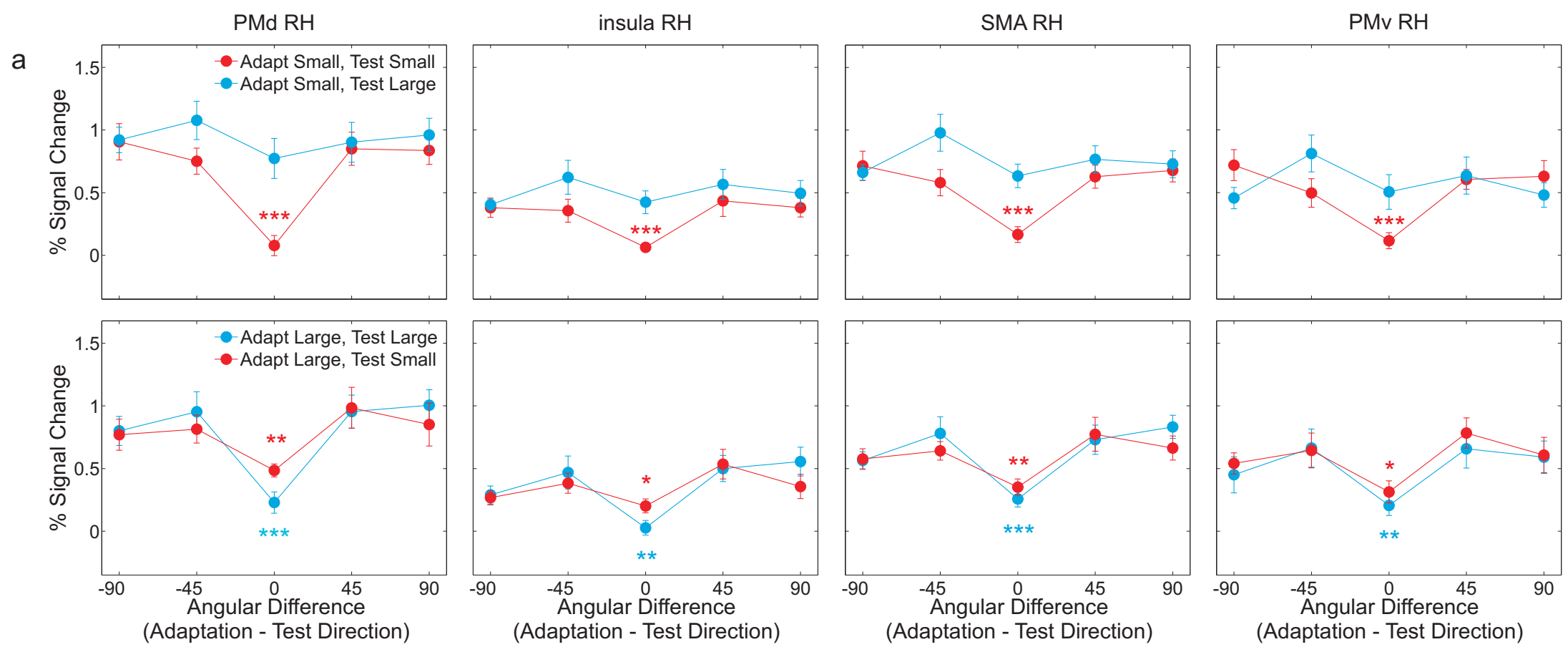

IPL RH
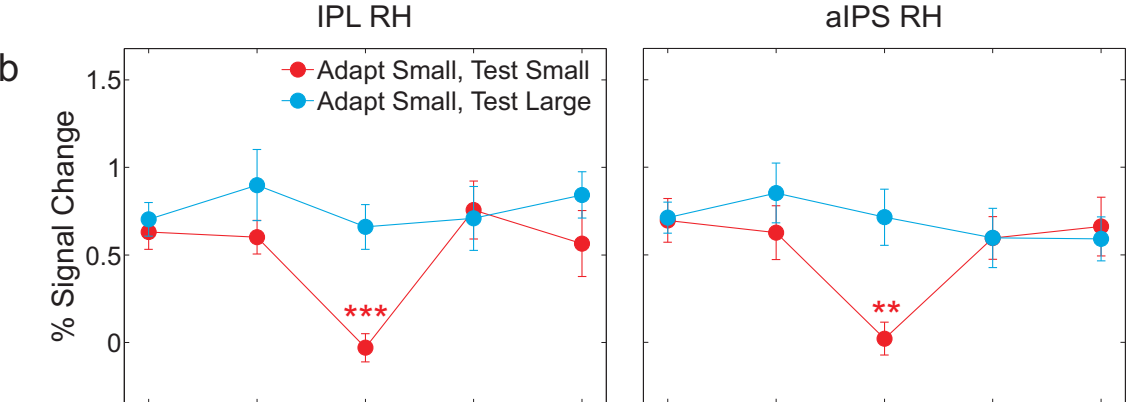

aPCu RH

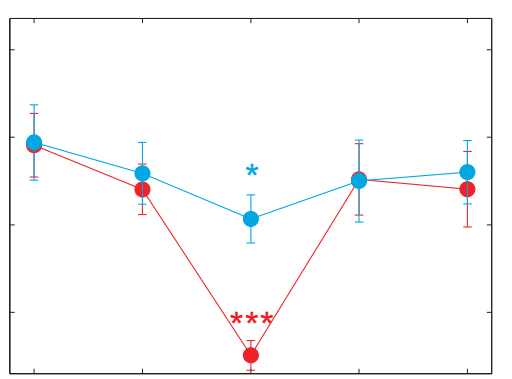

pIPS RH
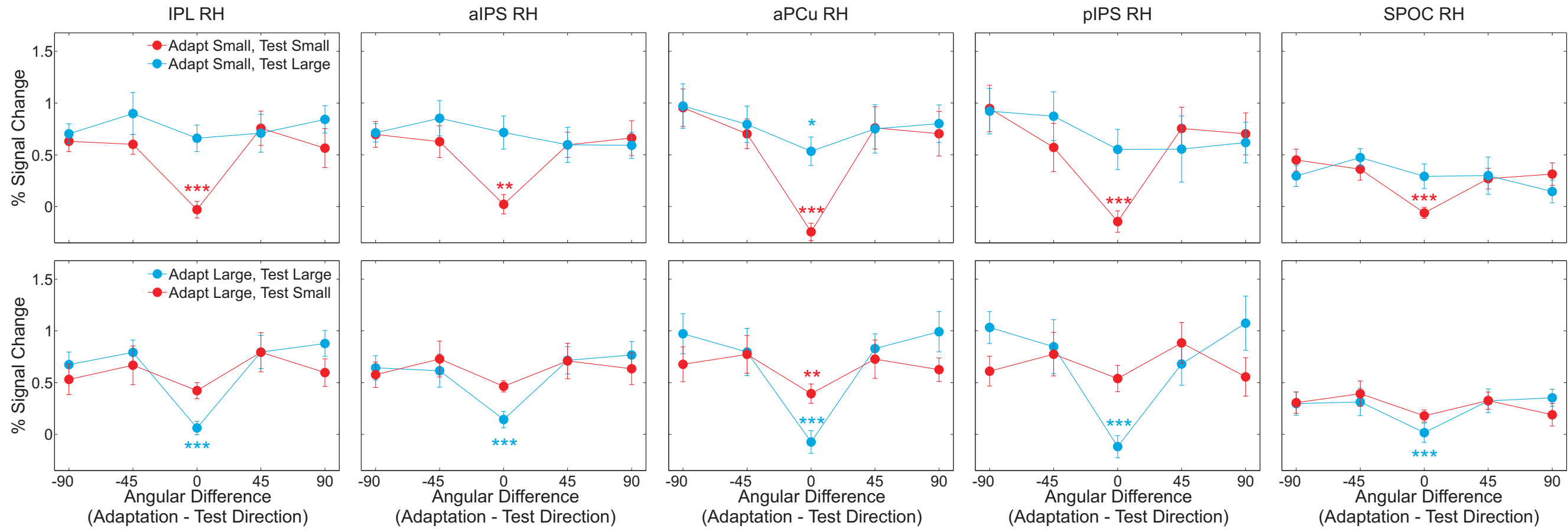

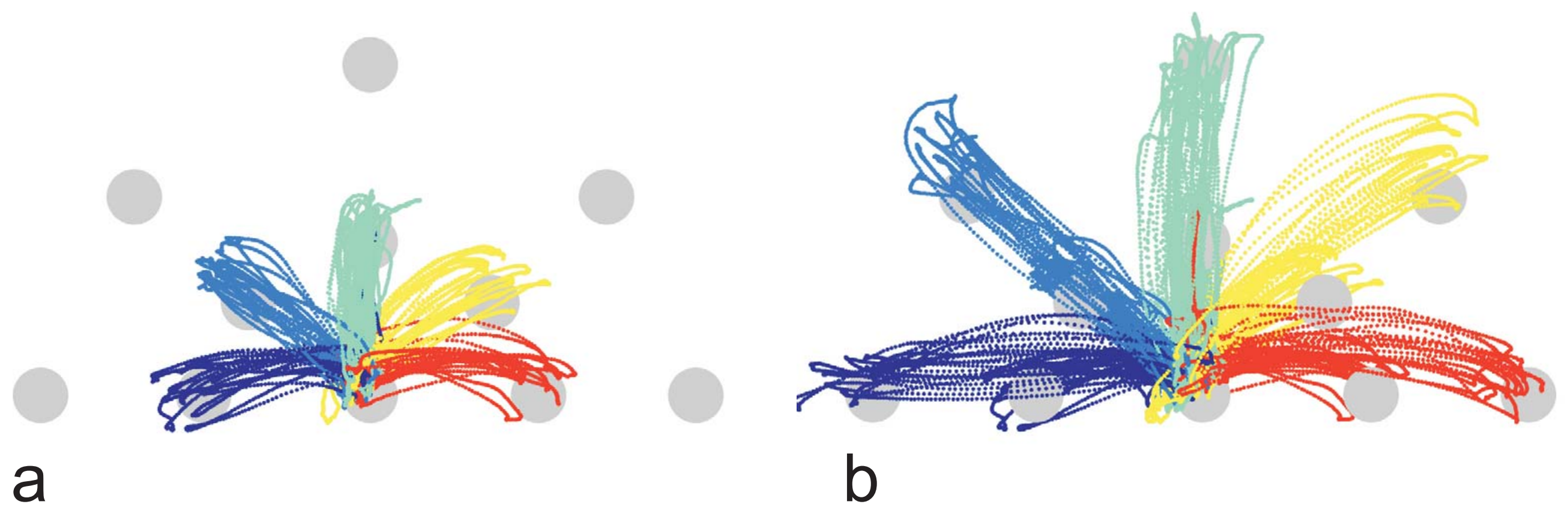

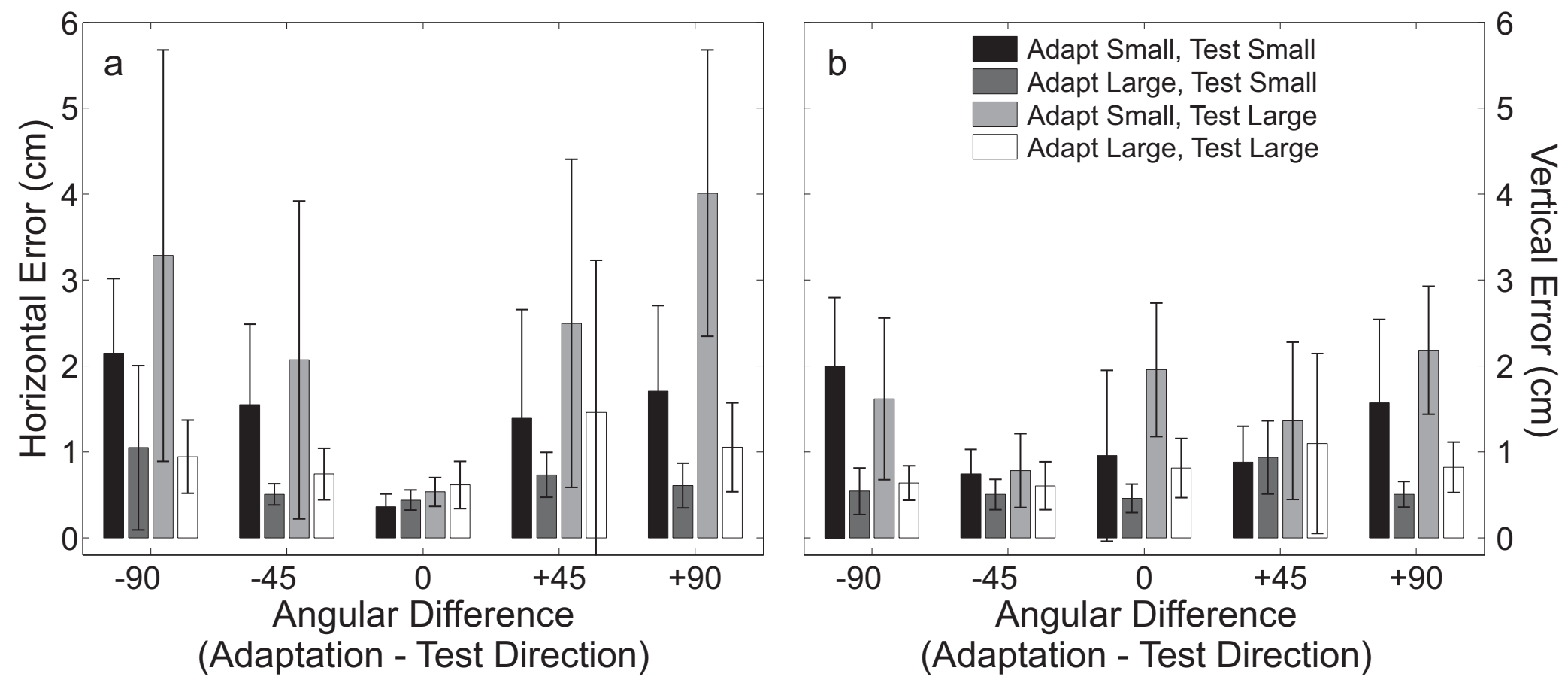


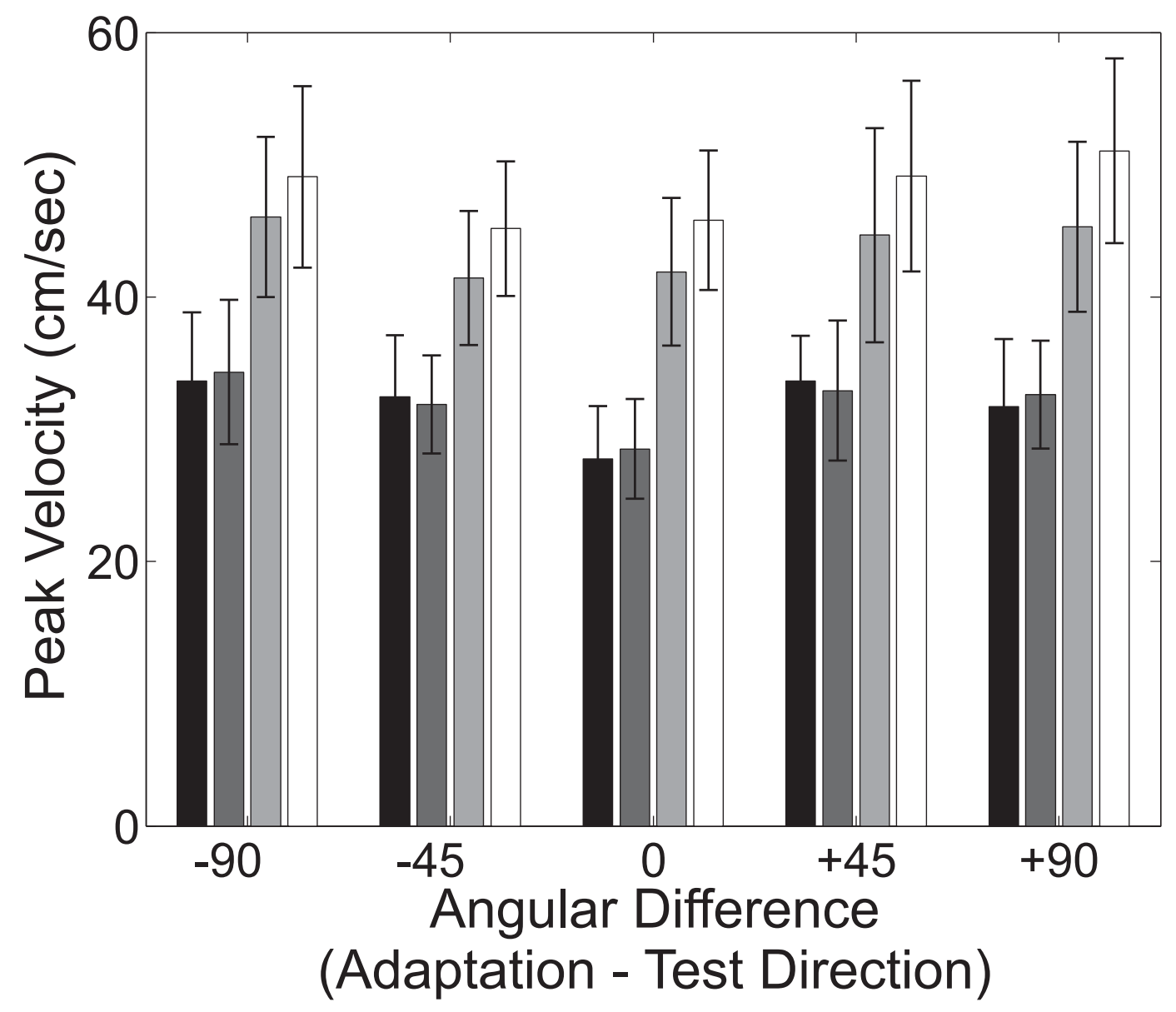

Adapt Small, Test Small Adapt Large, Test Small Adapt Small, Test Large Adapt Large, Test Large 
before adaptation

during adaptation

during test

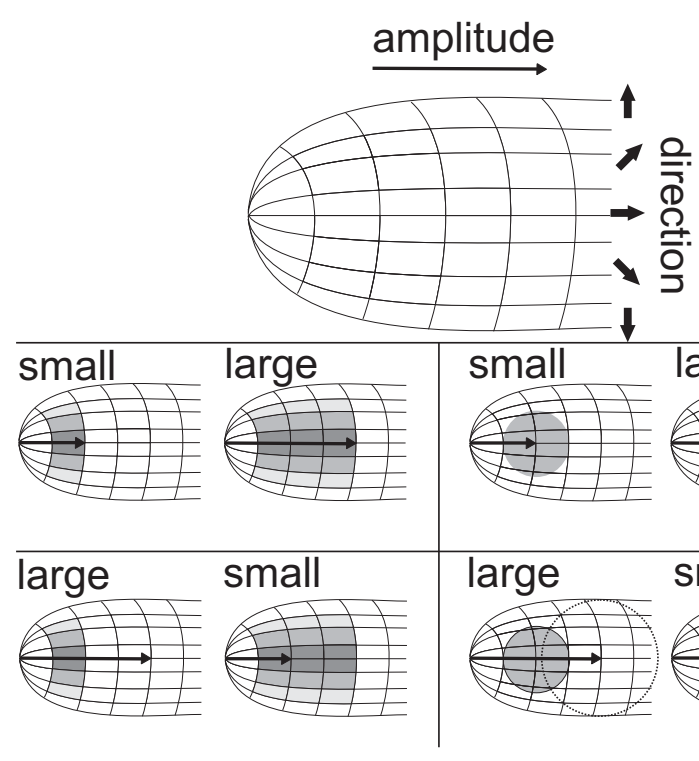

b

a response strength during small amplitude movements
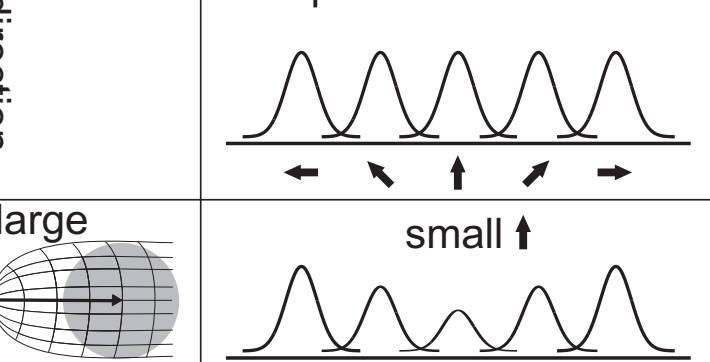

small

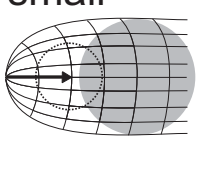

C
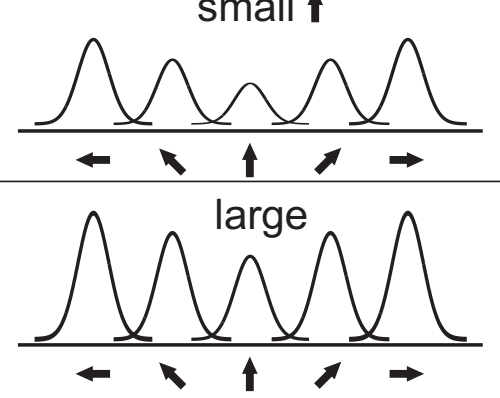

response strength during large amplitude movements
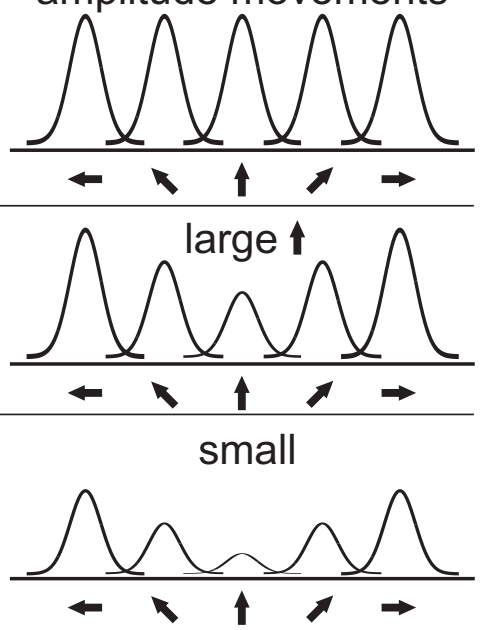


\begin{tabular}{lccc}
\hline & \multicolumn{3}{c}{ Talairach coordinates } \\
\cline { 2 - 4 } & $\mathrm{x}$ & $\mathrm{y}$ & $\mathrm{z}$ \\
\hline insula LH & -33.00 & 8.00 & 5.00 \\
insula RH & 31.00 & 6.00 & 6.00 \\
PMv LH & -48.00 & 0.00 & 24.00 \\
PMv RH & 41.00 & -3.00 & 36.00 \\
SMA LH & -9.00 & -7.00 & 46.00 \\
SMA RH & 8.00 & -2.00 & 50.00 \\
PMd LH & -27.00 & -16.00 & 56.00 \\
PMd RH & 24.00 & -11.00 & 53.00 \\
M1 LH & -33.00 & -32.00 & 53.00 \\
IPL LH & -53.00 & -33.00 & 31.00 \\
IPL RH & 52.00 & -33.00 & 33.00 \\
alPS LH & -35.00 & -44.00 & 40.00 \\
aIPS RH & 31.00 & -49.00 & 43.00 \\
aPCu RH & 11.00 & -63.00 & 53.00 \\
pIPS LH & -18.00 & -65.00 & 52.00 \\
pIPS RH & 10.00 & -73.00 & 45.00 \\
SPOC LH & -17.00 & -72.00 & 37.00 \\
SPOC RH & 21.00 & -73.00 & 31.00 \\
\hline Tab 1 & & &
\end{tabular}

Table 1 


\begin{tabular}{lcccccc}
\hline & \multicolumn{2}{c}{ Full Model } & \multicolumn{2}{c}{ Adapt Small } & \multicolumn{2}{c}{ Adapt Large } \\
\hline & $\begin{array}{c}\text { Interaction Adaptation } \\
\text { Amplitude } \\
\end{array}$ & \multicolumn{2}{c}{$\begin{array}{c}\text { x Direction } \\
\end{array}$} & $\begin{array}{c}\text { Interaction Test Amplitude } \\
\text { x Direction }\end{array}$ & $\begin{array}{c}\text { Interaction Test Amplitude } \\
\text { x Direction }\end{array}$ \\
\cline { 2 - 7 } & $\mathrm{F}(4,48)$ & $\mathrm{p}$ & $\mathrm{F}(4,48)$ & $\mathrm{p}$ & $\mathrm{F}(4,48)$ & $\mathrm{p}$ \\
\hline M1 LH & 2.786 & .037 & 3.869 & .008 & 2.131 & .091 \\
PMd LH & 4.179 & .006 & 4.355 & .004 & 2.267 & .076 \\
insula LH & 4.031 & .007 & 2.533 & .052 & 2.467 & .057 \\
SMA LH & 2.709 & .041 & 4.071 & .006 & 1.147 & .346 \\
PMv LH & 4.346 & .004 & 3.563 & .013 & 1.869 & .131 \\
IPL LH & 6.953 & $<.0001$ & 3.518 & .013 & 5.246 & .001 \\
alPS LH & 6.450 & $<.0001$ & 4.149 & .006 & 3.203 & .021 \\
pIPS LH & 7.853 & $<.0001$ & 1.996 & .110 & 5.960 & .001 \\
SPOC LH & 9.524 & $<.0001$ & 3.262 & .019 & 4.359 & .004 \\
\hline
\end{tabular}

Table 2 


\begin{tabular}{|c|c|c|c|c|c|c|}
\hline & \multicolumn{2}{|c|}{ Full Model } & \multicolumn{2}{|c|}{ Adapt Small } & \multicolumn{2}{|c|}{ Adapt Large } \\
\hline & \multicolumn{2}{|c|}{$\begin{array}{l}\text { Interaction Adaptation } \\
\text { Amplitude } x \text { Test } \\
\text { Amplitude } x \text { Direction }\end{array}$} & \multicolumn{2}{|c|}{$\begin{array}{c}\text { Interaction Test Amplitude } \\
\text { x Direction }\end{array}$} & \multicolumn{2}{|c|}{$\begin{array}{c}\text { Interaction Test Amplitude } \\
\text { x Direction }\end{array}$} \\
\hline & $F(4,48)$ & $p$ & $F(4,48)$ & $p$ & $F(4,48)$ & $p$ \\
\hline PMd RH & 6.272 & $<.0001$ & 4.669 & .003 & 1.736 & .158 \\
\hline insula RH & 2.358 & .067 & 1.797 & .145 & 1.409 & .245 \\
\hline SMA RH & 3.656 & .011 & 4.035 & .007 & 1.042 & .395 \\
\hline PMv RH & 2.555 & .051 & 5.461 & .001 & .189 & .943 \\
\hline IPL RH & 5.880 & .001 & 3.553 & .013 & 3.051 & .026 \\
\hline aIPS RH & 7.102 & $<.0001$ & 5.156 & .002 & 2.634 & .045 \\
\hline aPCu RH & 7.503 & $<.0001$ & 5.023 & .002 & 4.068 & .006 \\
\hline pIPS RH & 6.162 & $<.0001$ & 2.352 & .067 & 5.131 & .002 \\
\hline SPOC RH & 5.729 & .001 & 2.856 & .033 & 1.235 & .309 \\
\hline
\end{tabular}

Table 3 